\title{
Probabilistic analysis and design of strip foundations resting on rocks obeying Hoek-Brown failure criterion
}

\author{
Nut Mao, Tamara Al-Bittar, Abdul-Hamid Soubra \\ University of Nantes, Bd. de l'université, BP 152, 44603 Saint-Nazaire Cedex, France
}

\begin{abstract}
In this paper, a probabilistic analysis is presented to compute the probability density function (PDF) of the ultimate bearing capacity of a shallow strip footing resting on a rock mass. The rock is assumed to follow the modified Hoek-Brown failure criterion. Vertical and inclined loading cases are considered in the analysis. In this study, the deterministic models are based on the kinematic approach of the limit analysis theory. The polynomial chaos expansion (PCE) methodology is used for the probabilistic analysis. Four parameters related to the modified Hoek-Brown failure criterion are considered as random variables. These are the geological strength index (GSI), the uniaxial compressive strength of the intact rock $\left(\sigma_{c}\right)$, the intact rock material constant $\left(m_{i}\right)$ and the disturbance coefficient $(D)$. The results of the vertical load case have shown that (i) the variability of the ultimate bearing capacity increases with the increase in the coefficients of variation of the random variables; GSI and $\sigma_{c}$ being of greater effect, (ii) the non-normality of the input variables has a significant effect on the shape of the PDF of the ultimate bearing capacity, (iii) a negative correlation between GSI and $\sigma_{c}$ leads to less spread out PDF, (iv) the probabilistic footing breadth based on a reliability-based design (RBD) may be greater or smaller than the deterministic breadth depending on the values of the input statistical parameters. Finally, it was shown in the inclined load case that the variability of the ultimate bearing capacity decreases with the increase of the footing load inclination.
\end{abstract}

\section{Introduction}

Traditionally, stability analysis and design of shallow foundations resting on soils or rocks is based on deterministic approaches [1-4]. In this paper, the behavior of shallow foundations resting on a rock mass is studied using a probabilistic approach. The probabilistic approaches allow one to consider the propagation of the uncertainties from the input parameters to the system responses. Most probabilistic analyses existing in literature consider the ultimate limit state of foundations resting on a soil mass [5-12]. To the best of the authors' knowledge, there are no probabilistic studies on the ultimate limit state of shallow foundations resting on rock masses. The present paper fills this gap; it aims at determining the ultimate bearing capacity of a shallow strip footing resting on a rock mass using a probabilistic approach. The footing rests on a rock mass that follows the modified Hoek-Brown failure criterion. In this criterion, only intact rocks or heavily jointed rocks masses (i.e. with sufficiently dense and randomly distributed joints) can be considered. A central (vertical or inclined) footing load is considered in the analysis. The deterministic models are based on the kinematic

\footnotetext{
* Corresponding author. Tel.: +33 240905 108; fax: +33240905 109.

E-mail address: abed.soubra@univ-nantes.fr (A.-H. Soubra).
}

approach of limit analysis theory using translational multiblock failure mechanisms. Four uncertain parameters related to the modified Hoek-Brown failure criterion are modeled as random variables. These are the geological strength index (GSI), the uniaxial compressive strength of the intact rock $\left(\sigma_{c}\right)$, the intact rock material constant $\left(m_{i}\right)$ and the disturbance coefficient $(D)$. Notice that the system response considered in this paper is the ultimate bearing capacity of the footing. This response is related to the failure of the footing by rock punching.

As for the probabilistic studies, the classical Monte Carlo simulation (MCS) methodology is generally used to compute either the probability density function (PDF) of the system response or the failure probability $P_{f}$. In spite of being a rigorous and a robust methodology, MCS requires a great number of calls to the deterministic model (about 1,000,000 samples for a failure probability of $10^{-5}$ ). This is not convenient in case where the computation of the system response is not given by a simple analytical formula. In the present paper, a more efficient method based on the polynomial chaos expansion (PCE) is used [13-16]. The PCE methodology aims at replacing the deterministic model (for which the uncertain input parameters are modeled by random variables) by an approximate simple analytical equation called meta-model. Thus, one can easily calculate the system response when performing Monte Carlo simulations. In this paper, the meta-model is used to perform both 
probabilistic and reliability-based analyses. It is also used to perform a reliability-based design.

In the probabilistic analysis, a parametric study was performed. The PDF of the system response (ultimate bearing capacity) was determined for normal and non-normal random variables and for different values of the statistical parameters of the random variables (i.e. coefficient of variation and correlation between random variables). Also, a global sensitivity analysis based on Sobol indices was performed. These indices give the contribution of each random variable or combination of random variables in the variability of the system response. This is important because it helps engineers in detecting the uncertain parameters that have a significant influence in the variability of the system response. Concerning the reliabilitybased analysis, the meta-model determined by the probabilistic analysis was used to compute the Hasofer-Lind reliability index and the corresponding failure probability for different values of the applied footing load. In addition to the reliability-based analysis, a reliability-based design (RBD) was performed to determine (for given values of the statistical parameters of the input random variables) the footing breadth corresponding to a target failure probability.

The paper is organized as follows: The next section aims at presenting the basic idea of the polynomial chaos expansion (PCE) methodology. It is followed by a presentation of the deterministic model used for the computation of the ultimate bearing capacity of a centrally loaded strip footing (vertical or inclined load) resting on a rock mass. Finally, the probabilistic and reliability-based results are presented and discussed for both cases of vertical and inclined loadings. The paper ends with a conclusion.

\section{Polynomial chaos expansion (PCE) methodology}

The polynomial chaos expansion methodology allows one to represent by an analytical equation (meta-model) the response of a mechanical system whose input uncertain parameters are modeled by random variables. The main advantage of a PCE methodology is that the PDF of the system response can be easily obtained by applying MCS on the meta-model. Another important advantage of the meta-model is that it can be used to perform a global sensitivity analysis based on Sobol indices. These indices give the contribution of each random variable or combination of random variables in the variability of the system response. It should be noticed that the meta-model aims at presenting the random model response by a set of coefficients in a suitable (socalled polynomial chaos) basis. These coefficients may be efficiently computed using a non-intrusive technique where the deterministic model does not need to be modified; it is treated as a black box. Two non-intrusive approaches have been proposed in literature: the projection and the regression approach. In this paper, the regression approach [13-16] is used. Once the unknown coefficients of the PCE are determined, this PCE will be called "meta-model" and it will be used for further posttreatment in the probabilistic analysis. Thus, the PDF of the response can be derived with no cost since one makes use of the simple analytical formula. In this section, the main idea of the PCE methodology is first described. It is followed by a presentation of (i) the regression method used to determine the unknown PCE coefficients, (ii) the statistical analyses (determination of the PDF of the system response and the Sobol indices) by using the meta-model and (iii) the numerical implementation of the PCE methodology.

\subsection{System output}

Consider a deterministic model with $M$ input uncertain parameters gathered in a vector $X=\left\{X_{1}, \ldots, X_{M}\right\}$. The different elements
Table 1

Usual probability density functions and their corresponding families of orthogonal polynomials.

\begin{tabular}{ll}
\hline Probability density functions & Polynomials \\
\hline Gaussian & Hermite \\
Gamma & Laguerre \\
Beta & Jacobi \\
Uniform & Legendre \\
\hline
\end{tabular}

of this vector can have different types of the probability density function. In order to represent our mechanical system response by a PCE, all the uncertain parameters should be represented by a unique chosen PDF. Table 1 presents the usual probability density functions and their corresponding families of orthogonal polynomials. In this paper, the independent standard normal space is used. Thus, the suitable corresponding basis is the multidimensional Hermite polynomials. The expressions of the multi-dimensional Hermite polynomials are given in [16] among others.

Based on the Gaussian PDF chosen in this paper, Xiu and Karniadakis [17] have shown that the system response can be expanded onto an orthogonal polynomial basis as follows:

$\Gamma_{P C E}(\xi)=\sum_{\beta=0}^{\infty} a_{\beta} \Psi_{\beta}(\xi) \cong \sum_{\beta=0}^{P-1} a_{\beta} \Psi_{\beta}(\xi)$

where $\xi$ is the vector resulting from the transformation of the random vector $X$ into an independent standard normal space, $a_{\beta}$ are the unknown coefficients to be computed and $\Psi_{\beta}$ are the multidimensional Hermite polynomials. The PCE representation should be truncated by retaining only the multivariate polynomials of degree less than or equal to the PCE order $p$. This truncation scheme leads to a number $P$ of unknown coefficients given by

$P=\frac{(M+p) !}{M ! p !}$

For the determination of the PCE unknown coefficients, it is required to evaluate the system response at a set of collocation points (i.e. sampling points). As suggested by several authors [13-16], the roots of the one-dimensional Hermite polynomial (of one degree higher than the PCE order $p$ ) are used for each random variable. The collocation points are the result of all possible combinations of these roots. Thus, the number $N$ of the available collocation points depends on the number $M$ of the random variables and the PCE order $p$ as follows:

$N=(p+1)^{M}$

As proposed in [13-16], this number of collocation points should be increased by one when using a PCE of an odd order since in this case the number of collocation points does not include the origin. The origin should be included since it represents the point with the highest failure probability.

It should be mentioned here that in order to perform the deterministic calculations, one should transform the independent standard normal random variables of a given collocation point to the physical correlated non-normal space (if the physical variables are correlated and non-normal). This is done by first correlating the independent standard normal random variables of a given collocation point, by multiplying these independent standard variables by the Cholesky transform $\mathrm{CH}$ of the standard covariance matrix (i.e. correlation matrix) as follows:

$\xi^{c}=\mathrm{CH} \cdot \xi$

where $\xi^{c}$ is the correlated standard normal random vector and $\xi$ is the independent standard normal random vector. The standard correlated normal vector has now to be transformed 
into the non-normal space using the following equation:

$X=F^{-1}\left[\Phi\left(\xi^{c}\right)\right]$

where $X$ is the physical random vector, $F($.$) is the cumulative$ density function (CDF) in the non-normal space and $\Phi($.$) is the$ normal CDF. The next section is devoted to the presentation of the regression approach used to calculate the coefficients $a_{\beta}$ of the PCE.

\subsection{Regression approach}

As may be seen from Eq. (3), the number of the available collocation points dramatically increases as $p$ or $M$ increases. This number is always higher than the number $P$ of the unknown coefficients (given by Eq. (2)) when $M \geq 2$. This leads to a linear system of equations whose number of equations $N$ is greater than the number of unknowns $P$. Based on the regression approach, the vector of the unknown coefficients can be obtained by solving the following equation:

$a_{\beta}=\left(\Omega^{T} \Omega\right)^{-1} \Omega^{T} Y$

where $Y=\left\{Y^{1}, \ldots, Y^{N}\right\}$ is the vector of the model response values (computed via the deterministic model for the $N$ collocation points) and $\Omega$ is the matrix of dimensions $N \times P$. It is given by:

$\Omega=\left[\begin{array}{cccc}\psi_{0}^{1}(\xi) & \psi_{1}^{1}(\xi) & \cdots & \psi_{P-1}^{1}(\xi) \\ \psi_{0}^{2}(\xi) & \psi_{1}^{2}(\xi) & \cdots & \psi_{P-1}^{2}(\xi) \\ \vdots & \vdots & \ddots & \vdots \\ \psi_{0}^{N}(\xi) & \psi_{1}^{N}(\xi) & \cdots & \psi_{P-1}^{N}(\xi)\end{array}\right]$

Several attempts have been made in literature to select the most efficient collocation points among the $N$ available ones to reduce the number of calls of the deterministic model. Webster et al. [18] selected a number $K$ of collocation points among the $N$ available points based on the empirical equation $K=(P+1)$. Isukapalli et al. [13] proposed another empirical equation $K=2 P$. In both approaches, the collocation points are chosen to be the nearest ones to the origin of the standard space of random variables. More recently, Sudret [19] proposed a more rational methodology for the determination of the necessary number of collocation points. This method is used in this paper. It is based on the invertibility of the information matrix $A=\Omega^{T} \Omega$. It can be described by the following steps: (a) the $N$ available collocation points are ordered in a list according to increasing norm, (b) the information matrix $A$ is first constructed using the first $P$ collocation points of the ordered list, i.e. the $P$ collocation points that are the closest ones to the origin of the standard space of the random variables and finally (c) this matrix is successively increased by adding each time another collocation point from the ordered list until the matrix becomes invertible. This leads to a number $K$ of collocation points smaller than the number $N$ of the available collocation points.

It should be noticed here that the quality of the output approximation via a PCE closely depends on the PCE order $p$. Let us consider $K$ realizations $\left\{\xi^{(1)}=\left(\xi_{1}^{(1)}, \ldots, \xi_{M}^{(1)}\right), \ldots, \xi^{(K)}=\left(\xi_{1}^{(K)}, \ldots\right.\right.$, $\left.\left.\xi_{M}^{(K)}\right)\right\}$ of the standard normal random vector $\xi$, and note $\Gamma=\left\{\Gamma\left(\xi^{(1)}\right), \ldots, \Gamma\left(\xi^{(K)}\right)\right\}$ the corresponding values of the model response determined by deterministic calculations. To ensure a good fit between the meta-model and the true deterministic model (i.e. to obtain the optimal PCE order), the simplest error estimate is the well-known coefficient of determination $R^{2}$ given by:

$R^{2}=1-\frac{\Delta_{P C E}}{\operatorname{Var}(\Gamma)}$ where $\Delta_{P C E}$ is the empirical error given by:

$\Delta_{P C E}=\frac{1}{K} \sum_{i=1}^{K}\left[\Gamma\left(\xi^{(i)}\right)-\Gamma_{P C E}\left(\xi^{(i)}\right)\right]$

and

$\operatorname{Var}(\Gamma)=\frac{1}{K-1} \sum_{i=1}^{K}\left[\Gamma\left(\xi^{(i)}\right)-\bar{\Gamma}\right]^{2}$

$\bar{\Gamma}=\frac{1}{K} \sum_{i=1}^{K} \Gamma\left(\xi^{(i)}\right)$

The value $R^{2}=1$ indicates a perfect fit of the true model response $\Gamma$, whereas $R^{2}=0$ indicates a nonlinear relationship between the true model $\Gamma$ and the PCE model $\Gamma_{\text {PCE }}$. This coefficient may be a biased estimate since it does not take into account the robustness of the meta-model (i.e. its capability of correctly predicting the model response at any point which does not belong to the experimental design). As a consequence, one makes use of a more reliable and rigorous error estimate, namely the leave-one-out error estimate [20]. This error estimate consists in sequentially removing a point from the $K$ collocation points. Let $\Gamma_{\xi \backslash i}$ be the meta-model that has been built from the $K$ collocation points after removing the $i$ th observation from these collocation points and let $\Delta^{i}=\Gamma\left(\xi^{(i)}\right)-\Gamma_{\xi \backslash i}$ $\left(\xi^{(i)}\right)$ be the predicted residual between the model evaluation at point $\xi^{(i)}$ and its prediction based on $\Gamma_{\xi i}$. The empirical error is thus given as follows:

$\Delta_{P C E}^{*}=\frac{1}{K} \sum_{i=1}^{K}\left(\Delta^{i}\right)^{2}$

The corresponding coefficient of determination of the empirical error given by Eq. (12) is often denoted by $Q^{2}$ :

$Q^{2}=1-\frac{\Delta_{P C E}^{*}}{\operatorname{Var}[\Gamma]}$

\subsection{Statistical analysis}

Once the output approximation via a PCE is obtained, this PCE will be called meta-model and will be employed for the probabilistic and the reliability-based analyses. The PDF of the system response and the corresponding statistical moments (i.e. mean $\mu$, standard deviation $\sigma$, skewness $\delta$ and kurtosis $\kappa$ ) can be easily estimated. This can be done by simulating a large number of realizations of the standard normal variables on the meta-model using Monte Carlo technique. Another important advantage of the meta-model is that it can be used to perform a global sensitivity analysis (GSA). The GSA is generally based on the decomposition of the response variance as a sum of contributions of the different random variables or combinations of random variables (the sum of all Sobol indices is equal to 1 ). In this framework, Sobol indices give the contribution of each random variable or combination of random variables to the variability of the system response [19]. This is important because it helps engineers in detecting the uncertain input parameters which have a significant influence in the variability of the system response.

\subsection{Numerical implementation of the PCE methodology}

For the implementation of the PCE methodology, a computer program was developed in the commercial software Matlab 7.6. For each input random variable, the code first computes the roots of the one-dimensional Hermite polynomial (of one degree higher than the prescribed PCE order $p$ ) and then it provides the collocation points in the standard space of normal random variables. In a second 
step, the program uses isoprobabilistic transformation and Cholesky transformation of the standard covariance matrix to transform the collocation points to the corresponding physical space (in case of non-normal and correlated variables) in order to calculate the corresponding system response(s) using the deterministic model. Finally, the program computes the unknown coefficients of the PCE using the regression approach presented above. A probabilistic analysis can thus be performed using this code. The PDF of the system response and the corresponding statistical moments can be obtained by simulating a large number of standard normal random variables on the meta-model using the Monte Carlo technique. The Sobol indices for each random variable or a combination of random variables can also be determined using the coefficients of the PCE. On the other hand, the program can also be employed to perform a reliability analysis on the meta-model. This can be done easily since the obtained PCE is given in the standard space of the normal uncorrelated random variables. Thus, one can determine the reliability index and the corresponding design point for different values of the applied footing load. A reliability-based design based on the meta-model can also be performed to obtain the footing breadth for a target reliability index.

\section{Deterministic bearing capacity of a centrally loaded strip footing}

In this section, one first presents a brief description of the modified Hoek-Brown failure criterion. This is followed by a presentation of the two deterministic models used to compute the ultimate bearing capacity of a centrally loaded shallow strip footing (i.e. a footing subjected to a vertical or an inclined load).

\subsection{Modified Hoek-Brown failure criterion}

The modified Hoek-Brown failure criterion only deals with intact rocks or heavily jointed rock masses. A heavily jointed rock mass involves sufficiently dense and randomly distributed joints so that in the scale of the problem, it can be regarded as an isotropic assembly of interlocking particles. Consequently, rocks with few discontinuities cannot be considered in this framework [21-23]. The modified Hoek-Brown failure criterion can be described by the following equation [24]:

$\sigma_{1}-\sigma_{3}=\sigma_{c}\left(m \frac{\sigma_{3}}{\sigma_{c}}+s\right)^{n}$

where $\sigma_{1}$ and $\sigma_{3}$ are, respectively, the major and minor principal stresses at failure and $\sigma_{c}$ is the uniaxial compressive strength of the intact rock material. The parameters $m, s$ and $n$ are given by the following equations:

$m=m_{i} \exp \left(\frac{G S I-100}{28-14 D}\right)$

$s=\exp \left(\frac{G S I-100}{9-3 D}\right)$

$n=\frac{1}{2}+\frac{1}{6}\left[\exp \left(-\frac{G S I}{15}\right)-\exp \left(-\frac{20}{3}\right)\right]$

In these equations, the geological strength index (GSI) characterizes the quality of the rock mass and depends on its structure and its joints surface conditions [25]. On the other hand, the parameter $m_{i}$ is the value of parameter $m$ for intact rock and can be obtained from experimental tests. The parameter $m_{i}$ varies from 4 for very fine weak rock like claystone to 33 for coarse igneous light-colored rock like granite. Finally, $D$ is the disturbance coefficient. It varies from 0.0 for undisturbed in situ rock masses to 1.0 for very disturbed rock masses. Fig. 1 presents the

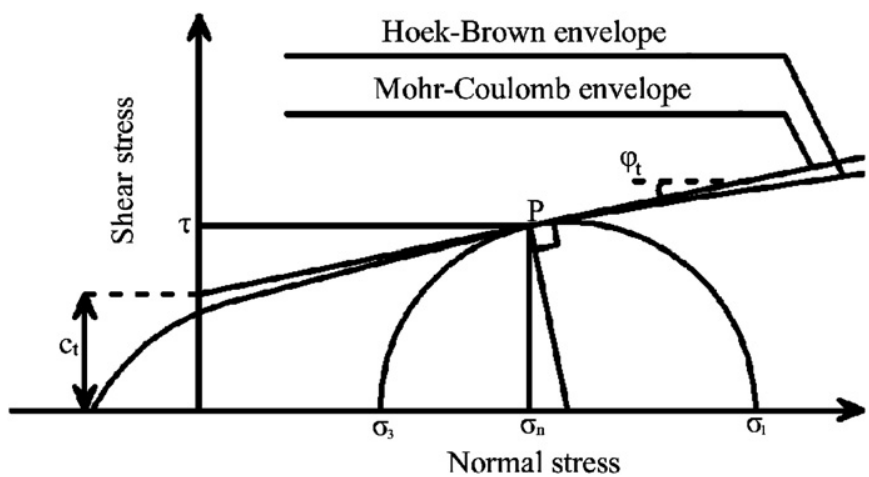

Fig. 1. Failure envelope of the Mohr-Coulomb and the Hoek-Brown failure criterion in the $(\sigma, \tau)$ plan.

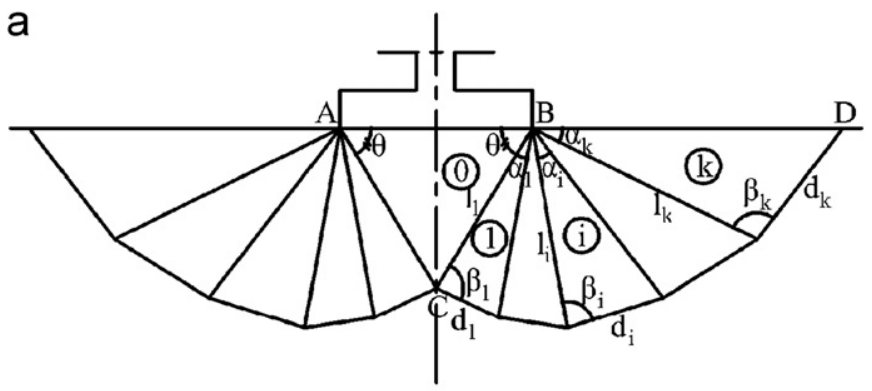

b

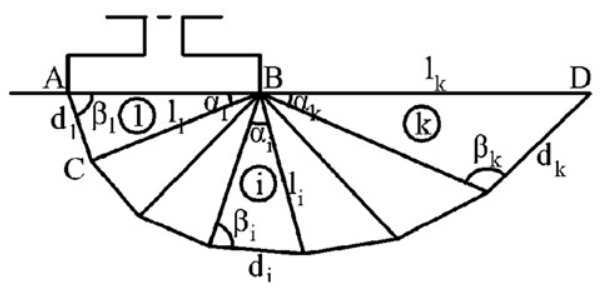

Fig. 2. Failure mechanisms for the computation of the ultimate bearing capacity of (a) vertically loaded foundation (M1 mechanism) and (b) obliquely loaded foundation (M2 mechanism)

nonlinear modified Hoek-Brown failure criterion in the $(\tau, \sigma)$ plane.

\subsection{Limit analysis models}

Two kinematically admissible failure mechanisms M1 and M2 based on the upper-bound theorem of limit analysis are used herein. These mechanisms were firstly presented by Soubra [1] for the computation of the ultimate bearing capacity of a strip footing resting on a soil mass. Later on, the M1 mechanism was used by Yang and Yin [3] and Saada et al. [4] for the case of a strip footing resting on a rock mass obeying Hoek-Brown failure criterion.

M1 is a translational symmetrical multiblock failure mechanism (Fig. 2a) and is used for the computation of the ultimate bearing capacity of a vertically loaded strip footing. It is composed of $2 k+1$ triangular rigid blocks (a central symmetrical block under the footing and $2 k$ symmetrical rigid blocks at both sides of the footing). This mechanism can be completely described by $2 k$ angular parameters which are $\alpha_{i}(i=1, \ldots, k-1), \beta_{i}(i=1, \ldots, k)$ and $\theta$. On the other hand, M2 is a translational non-symmetrical multiblock failure mechanism (Fig. 2b) and is suitable for the calculation of the ultimate bearing capacity of an obliquely loaded strip foundation. This mechanism is composed of $k$ triangular rigid blocks. It can be completely described by $2 k-1$ angular 
parameters which are $\alpha_{i}(i=1, \ldots, k-1)$ and $\beta_{i}(i=1, \ldots, k)$. The computation of the ultimate bearing capacity is performed by equating the total rate of work of the external forces $\dot{W}$ to the total rate of energy dissipation $\dot{D}$ along the lines of velocity discontinuities. For more details on these mechanisms, the reader can refer to [1].

The above mechanisms were used by Soubra [1] in the case of a Mohr-Coulomb material (i.e. a soil mass) where the failure envelope is linear. For a rock mass obeying the modified Hoek-Brown failure criterion, the failure envelope is nonlinear (see Fig. 1). Yang and Yin [3] replaced the nonlinear modified Hoek-Brown failure criterion by a linear Mohr-Coulomb failure criterion represented by a tangential line (see Fig. 1) where $P$ is the tangent point. This criterion is given by

$\tau=c_{t}+\sigma_{n} \tan \varphi_{t}$

where $\varphi_{t}$ is the tangential friction angle and $c_{t}$ is the intercept of the tangential line with the $\tau$-axis in the $(\tau, \sigma)$ plan. This technique has also been used in [26] among others. For the tangent point $P$, the cohesion $c_{t}$ can be expressed in terms of (i) the tangential friction angle $\varphi_{t}$ and (ii) the parameters $m, s, n$ of the Hoek-Brown failure criterion as follows:

$$
\begin{aligned}
\frac{c_{t}}{\sigma_{c}}= & \frac{\cos \varphi_{t}}{2}\left[\frac{m n\left(1-\sin \varphi_{t}\right)}{2 \sin \varphi_{t}}\right]^{(1 /(1-n))} \\
& -\frac{\tan \varphi_{t}}{m}\left(1+\frac{\sin \varphi_{t}}{n}\right)\left[\frac{m n\left(1-\sin \varphi_{t}\right)}{2 \sin \varphi_{t}}\right]^{(1 /(1-n))}+\frac{s}{m} \tan \varphi_{t}
\end{aligned}
$$

Notice that the location of the tangent point $P$ is obtained by optimization as will be shown later. By using the tangential line method, the rate of energy dissipation per unit area along a given velocity discontinuity surface remains essentially the same as that of the linear Mohr-Coulomb criterion but with $c_{t}$ and $\varphi_{t}$ instead of $c$ and $\varphi$ as follows:

$\dot{D}=c_{t} v \cdot \cos \varphi_{t}$

where $v$ is the velocity along a given velocity discontinuity surface. Notice that in this approach, all the block velocities $v_{i}$ and all the inter-block velocities $v_{i, i+1}$ are assumed to be inclined at a constant angle $\varphi_{t}$ (the tangential friction angle) with respect to their corresponding velocity discontinuity surfaces as shown in Fig. 3a for the case of a symmetrical mechanism. Since the strength given by the tangential line (for a given value of the normal stress) is either equal or exceeds that of the nonlinear failure criterion (see Fig. 1), the solution obtained using the tangential line method is certainly greater than that of a nonlinear failure criterion and thus, it remains a strict upper-bound to the exact solution.

Recently, a more rigorous and efficient approach, which preserves the original nonlinear form of the modified Hoek-Brown failure criterion, was proposed by Saada et al. [4]. Contrary to the tangential line method where a single tangential friction angle $\varphi_{t}$ was used; in the method by Sadaa et al. [4], each wedge $i(i=1, \ldots, k)$ is assumed to move with a velocity $v_{i}(i=1, \ldots, k)$ inclined at angle $\varphi_{i}$ with respect to line $d_{i}$. As for the relative velocity $v_{i, i+1}$, it was arbitrarily considered to be inclined at the same angle as that of line $d_{i+1}$ (i.e. $\varphi_{i, i+1}=\varphi_{i+1}$ ) (Fig. $3 \mathrm{~b}$ ). Although the approach by Saada et al. [4] constitutes a significant improvement with respect to the method by Yang and Yin [3] (since these authors make use of several tangential friction angles and not only of a single tangential friction angle everywhere in the rock mass), their assumption concerning the inclination of the relative velocity $v_{i, i+1}$ is a shortcoming. This shortcoming will be removed in the present paper. Thus, the velocity $v_{i, i+1}$ will be assumed herein as being inclined at an angle $\varphi_{i, i+1}$ to line $l_{i+1}$ where $\varphi_{i, i+1}$ will be different along the different lines $l_{i+1}$ (Fig. 3c). By using this
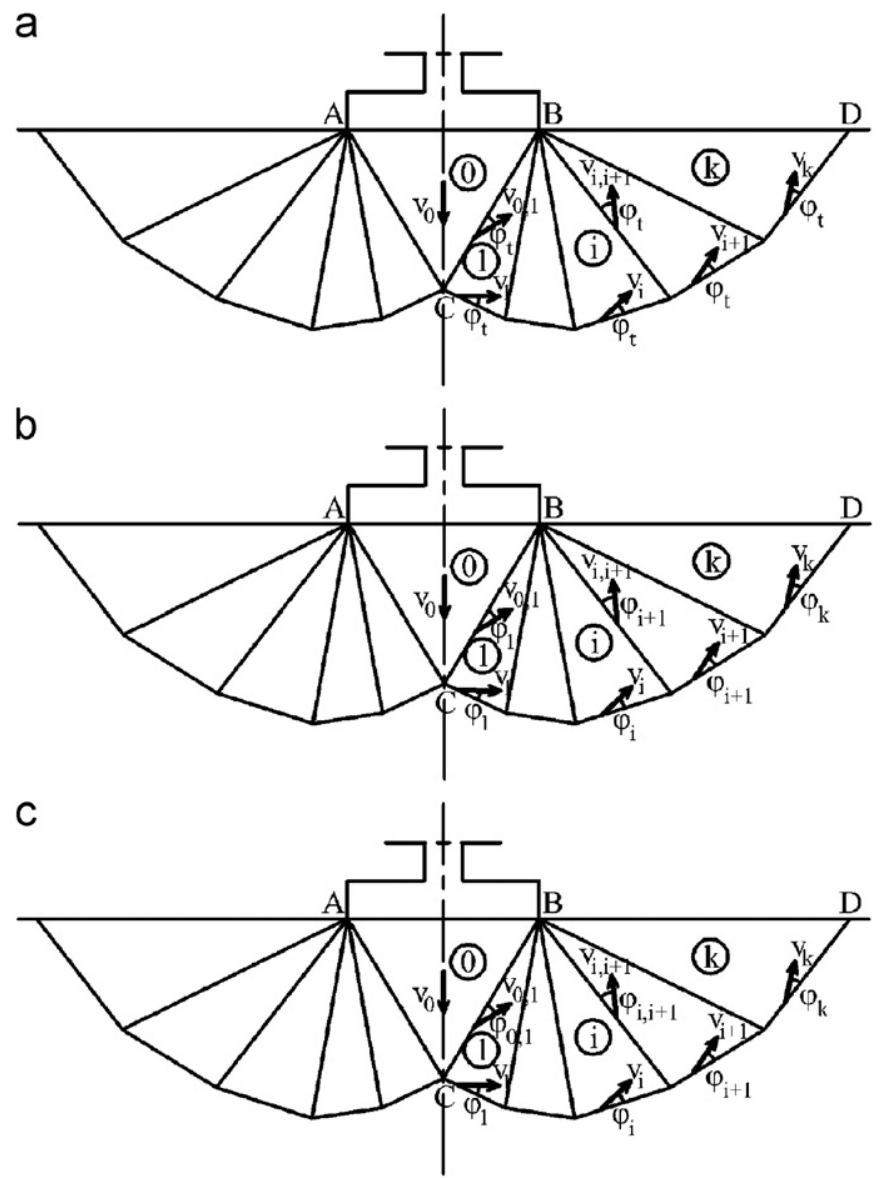

Fig. 3. Velocity field of M1 mechanism as given by (a) Yang and Yin [3]; (b) Saada et al. [4]; (c) present approach.

approach, a higher number of degree of freedom will be added to the failure mechanism.

Notice that the numerical results have shown that the increase in the number of blocks decreases (i.e. improves) the ultimate bearing capacity. However, the increase from seven blocks to eight blocks decreases (i.e. improves) the solution by a small percentage $(<0.8 \%)$. Thus only seven blocks will be used hereafter. Notice also that the improvement induced by the present approach with respect to that by Saada et al. [4] (where $\varphi_{i, i+1}=\varphi_{i+1}$ ) was found equal to $0.9 \%$ for seven blocks. Although, this improvement is very small, the present approach will be used hereafter with seven rigid blocks, the increase in the computation time with the present approach being not significant (only few seconds). It should be emphasized here that by using several tangential friction angles (as is the case in the approach of [4] and by the present approach), the modified HoekBrown failure criterion will be implicitly represented by a series of tangential lines to the nonlinear failure criterion. The rate of energy dissipation $\dot{D}$ used in this case is given as follows [4]:

$\dot{D}=\frac{s \sigma_{c}}{m} v^{(n)}+\sigma_{c}\left(n^{(n /(n-1))}-n^{(1 /(n-1))}\right) m^{(n /(n-1))}\left(\frac{1}{v^{(n)}}\left(\frac{v-v^{(n)}}{2}\right)^{1 / n}\right)^{(n /(n-1))}$

where $v^{(n)}$ is the normal component of a velocity $v$ along a given velocity discontinuity surface.

For both M1 and M2 mechanisms, it was found, after some simplifications, that an upper-bound of the ultimate bearing capacity is given by

$q_{u}=\frac{1}{2} \gamma B_{0} N_{\gamma}+q N_{q}+\sigma_{c} N_{\sigma_{c}}$ 
where $N_{\gamma}, N_{q}$ and $N_{\sigma_{c}}$ are non-dimensional parameters. They can be expressed in terms of (i) the mechanism geometrical parameters and (ii) the different tangential friction angles $\varphi_{i+1}$ and $\varphi_{i, i+1}$. These nondimensional parameters are given in Appendices $\mathrm{A}$ and $\mathrm{B}$ for both the M1 and M2 failure mechanisms, respectively. For each failure mechanism, the ultimate bearing capacity is obtained by minimization with respect to the angular parameters of the failure mechanism and with respect to the different tangential friction angles $\varphi_{i+1}$ and $\varphi_{i, i+1}$ of that failure mechanism.

\section{Probabilistic and reliability-based numerical results}

A strip footing of width $B_{0}=1 \mathrm{~m}$ placed on a weightless $(\gamma=0)$ rock mass, with no surcharge loading on the ground surface $(q=0)$ is considered in the analysis. As mentioned before, the four parameters $\left(G S I, \sigma_{c}, m_{i}, D\right)$ related to the modified Hoek-Brown failure criterion are considered as random variables. In order to incorporate the possible dependence between the parameters GSI and $\sigma_{c}$, a correlation coefficient was considered herein. In this paper, the illustrative values used for the coefficient of correlation and for the statistical moments of the different random variables are given in Table 2. However, other values of these parameters were considered in the framework of the parametric study. It should be mentioned here that the values of the statistical parameters of GSI, $m_{i}$ and $\sigma_{c}$ were proposed in [27]. As for the disturbance coefficient $D$, the practical values used in rock engineering problems are within a range of $0-0.6[3,4,24]$. Since there is no information about the coefficient of variation of the disturbance coefficient $D$ and the correlation coefficient, the illustrative values presented in Table 2 were adopted in this study. The deterministic models are based on the two failure mechanisms M1 and M2. The results are presented first for the case of a vertically loaded footing and then for the case of an obliquely loaded footing.

\subsection{Case of a vertically loaded footing}

\subsubsection{Optimal PCE order and Sobol indices}

This section aims at determining the optimal PCE order $p$ and the Sobol indices. To determine the optimal PCE order $p$, two alternative methods are used herein. The first one aims at determining the two coefficients of determination $R^{2}$ and $Q^{2}$ given respectively by Eqs. (8) and (13) although the computation of only $Q^{2}$ is sufficient since it is more restrictive than $R^{2}$. As mentioned before, a value of $R^{2}$ and $Q^{2}$ close to one indicates a good fit between the meta-model and the true model. The coefficients of determination $R^{2}$ and $Q^{2}$ were calculated for the different PCE orders (i.e. PCEs of order 2, 3, 4 and 5). Table 3 shows that $R^{2}$ and $Q^{2}$ of order 4 and order 5 are close to 1 (higher than 0.99999 ). Consequently, a PCE of order $p=4$ provides a good fit between the meta-model and the true model. The second method consists in verifying the two following conditions: (i) the absolute difference between the coefficients corresponding to the same terms in two PCEs of successive orders ( $p$ and $p+1)$ becomes smaller than a prescribed tolerance $\left(\varepsilon \leq 10^{-3}\right.$ for example) and (ii) the coefficients of the new terms of the PCE of order $p+1$ tend to be negligible. The numerical results (results not provided herein) have shown that the absolute differences between the coefficients of the common terms of the PCEs of order 4 and 5 are less than $10^{-3}$. These results have also shown that the coefficients of the new terms in the PCE of order 5 are all less than $10^{-3}$. These two observations confirm the adequacy of the choice done (i.e. $p=4$ ) with the method based on the coefficients of determination. Thus, a PCE of order $p=4$ will be used for all probabilistic calculations performed in this paper.

It should be mentioned here that the above two methods allow one to check the adequacy of the PCE order for the accurate computation of the statistical moments of the system response. To check the adequacy of the PCE order at the tail distribution (for the computation of the failure probability), Fig. 4 presents the CDF of the ultimate bearing capacity provided by the PCEs of orders $2,3,4$, 5 and 6. From this figure, one can see that the PCEs of orders 4, 5 and 6 can be considered as adequate since there is no difference between the CDFs of these PCEs in the zone of small failure probability. Notice that the distance from the origin of the standard space that is covered by the sampling zone is equal to 3.1623, 3.4917 and 3.9241 for the PCEs of orders 4, 5 and 6, respectively. Since the reliability

Table 3

Coefficients of determination $R^{2}$ and $Q^{2}$ of the different PCE orders.

\begin{tabular}{lll}
\hline Order of PCE & \multicolumn{2}{l}{ Coefficients of determination } \\
\cline { 2 - 3 } & $R^{2}$ & $Q^{2}$ \\
\hline 2 & 0.9990050287 & 0.9956879877 \\
3 & 0.9999903833 & 0.9996685645 \\
4 & 0.9999996619 & 0.9999977432 \\
5 & 0.9999999945 & 0.9999999791 \\
\hline
\end{tabular}

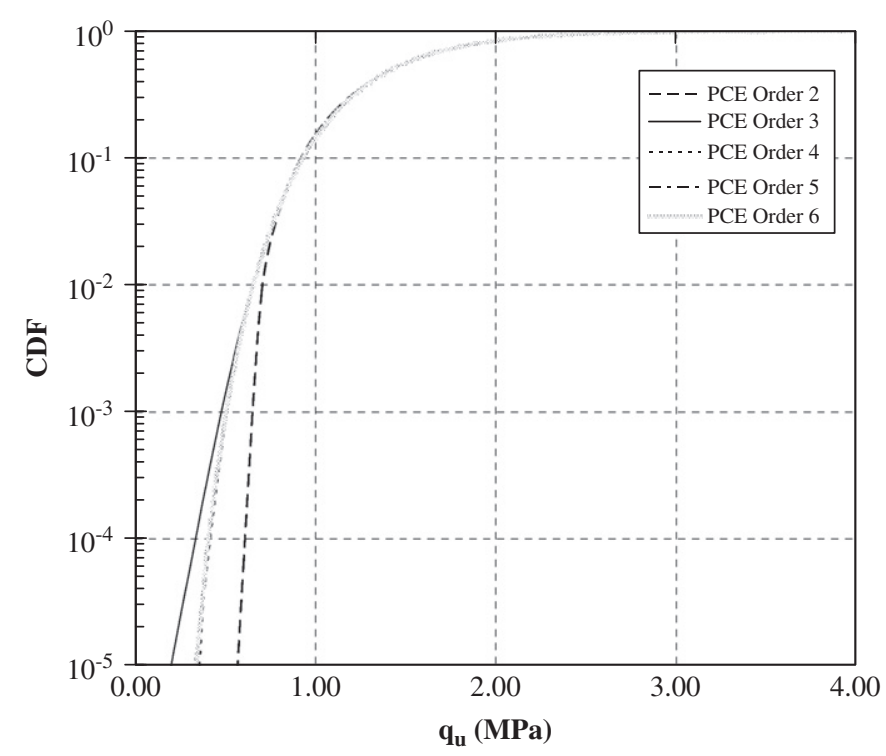

Fig. 4. Influence of the PCE order on the CDF of the ultimate bearing capacity.

Table 2

Input random variables and their statistical characteristics.

\begin{tabular}{lclll}
\hline Variables & Mean $\mu$ & $\begin{array}{l}\text { Coefficient of } \\
\text { variation } \operatorname{COV}(\%)\end{array}$ & $\begin{array}{l}\text { Type of the probability } \\
\text { density function }\end{array}$ & $\begin{array}{l}\text { Coefficient of } \\
\text { correlation } \rho\end{array}$ \\
\hline$G S I[-]$ & 25 & 10 & Log-normal & $-0.75 \leq \rho\left(G S I, \sigma_{c}\right) \leq+0.75$ \\
$m_{i}[-]$ & 8 & 12.5 & Log-normal & Log-normal \\
$\sigma_{c}[\mathrm{MPa}]$ & 10 & 25 & Log-normal & \\
$D[-]$ & 0.3 & 10 &
\end{tabular}


index used in the (RBD) is equal to 3.8 (as will be shown later), a PCE of order 6 is thus necessary because the corresponding sampling zone covers a distance larger than 3.8. Thus, the reliability analysis

Table 4

Sobol' indices for a fourth order PCE.

\begin{tabular}{ll}
\hline$S U(G S I)$ & 0.3141 \\
$S U\left(m_{i}\right)$ & 0.0866 \\
$S U\left(\sigma_{c}\right)$ & 0.5378 \\
$S U(D)$ & 0.0308 \\
$S U\left(G S I, m_{i}\right)$ & $2.62 \times 10^{-3}$ \\
$S U\left(G S I, \sigma_{c}\right)$ & 0.0196 \\
$S U(G S I, D)$ & $5.91 \times 10^{-4}$ \\
$S U\left(m_{i}, \sigma_{c}\right)$ & $5.41 \times 10^{-3}$ \\
$S U\left(m_{i}, D\right)$ & $3.23 \times 10^{-4}$ \\
$S U\left(\sigma_{c}, D\right)$ & $1.92 \times 10^{-3}$ \\
$S U\left(G S I, m_{i}, \sigma_{c}\right)$ & $1.56 \times 10^{-3}$ \\
$S U\left(G S I, m_{i}, D\right)$ & $4.71 \times 10^{-6}$ \\
$S U\left(G S I, \sigma_{c}, D\right)$ & $3.57 \times 10^{-5}$ \\
$S U\left(m_{i}, \sigma_{c}, D\right)$ & $1.97 \times 10^{-5}$ \\
$S U\left(G S I, m_{i}, \sigma_{c}, D\right)$ & $2.70 \times 10^{-7}$ \\
$S u m m a t i o n$ & 1.0000
\end{tabular}

a

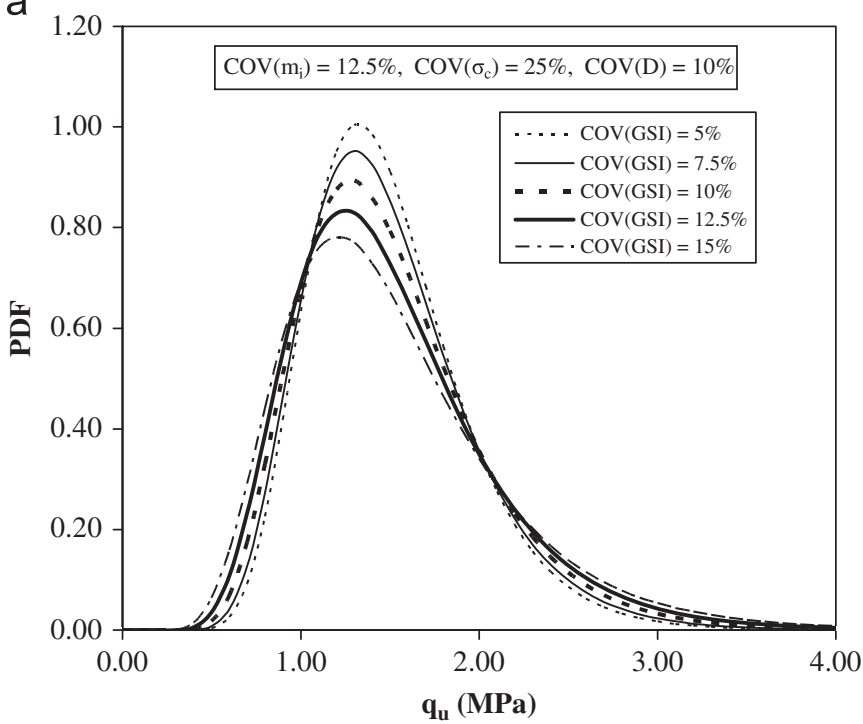

C

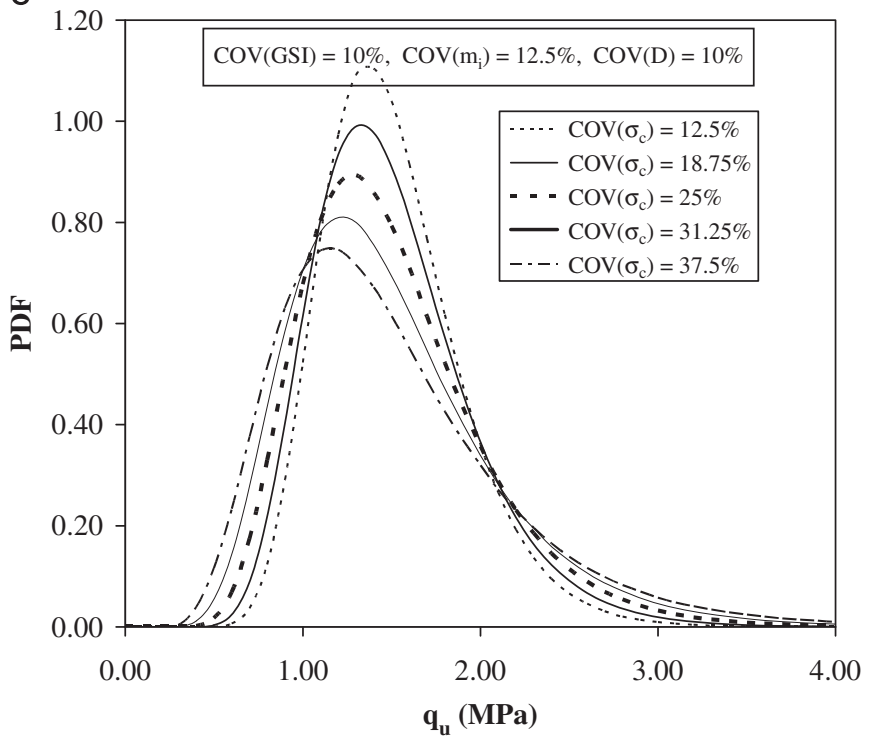

will be performed using a PCE of order 6 while all the probabilistic analyses will be performed using a PCE of order 4 .

Table 4 presents the Sobol indices (denoted by $S U$ ) of the different random variables or combinations of random variables obtained using a fourth order PCE. As mentioned previously, the Sobol indices provide the contribution of each random variable or a combination of random variables to the response variability. From Table 4 , one can observe that the Sobol index of the parameter $\sigma_{c}$ is higher than that of all the other parameters. Consequently, $\sigma_{c}$ has the most important contribution in the variability of the system response (i.e. the ultimate bearing capacity). Another influencing parameter is GSI which has a Sobol index of 0.3141. As for the two remaining parameters (i.e. $m_{i}$ and $D$ ), their contribution is less important due to the small values of their Sobol indices. Also, the Sobol indices of all combinations of random variables are negligible. This study is important because it helps engineers in detecting the uncertain parameters which have a significant weight in the variability of the system response. For a given rock mass, a thorough experimental investigation on the variability of the input parameters will thus be required by the engineer for only the influential parameters [i.e. the geological strength index (GSI) and the uniaxial

b

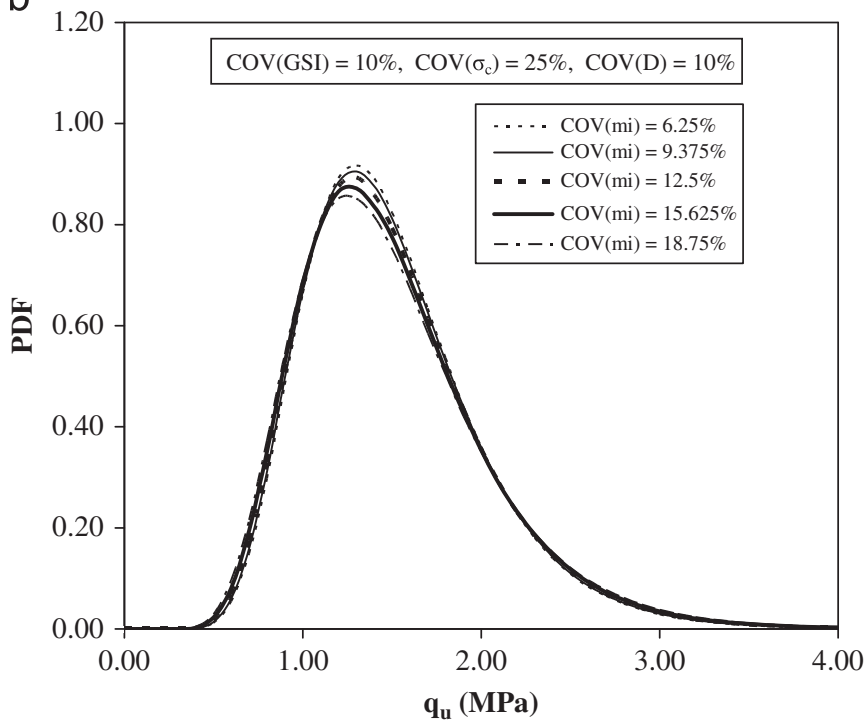

d

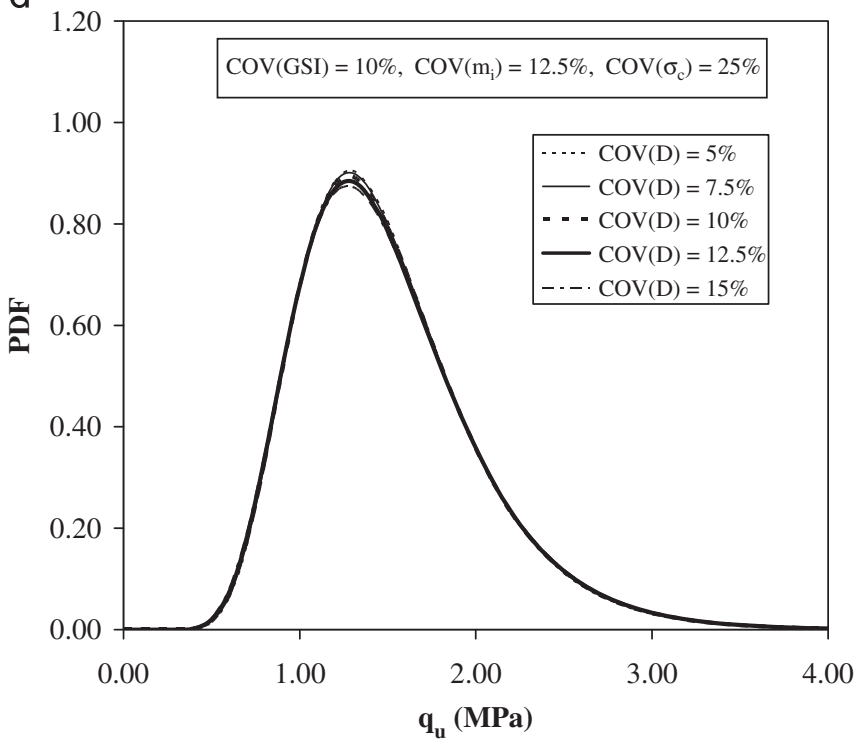

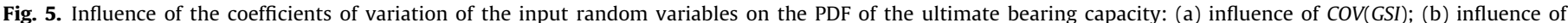
$\operatorname{COV}(m i)$; (c) influence of $\operatorname{COV}\left(\sigma_{c}\right)$; (d) influence of $\operatorname{COV}(D)$. 
Table 5

Effect of the coefficients of variation of the input random variables on the statistical moments of the ultimate bearing capacity.

\begin{tabular}{lllllll}
\hline & $\begin{array}{l}\text { Coefficient } \\
\text { of variation } \\
\%\end{array}$ & $\mu$ & $\sigma$ & COV\% & $\delta$ & $\begin{array}{l}\text { Deterministic } \\
\text { value of } q_{u}\end{array}$ \\
& $\%$ & & & & & \\
& 5 & 1.5037 & 0.4395 & 29.4 & 0.9017 & 1.4701 \\
$\operatorname{COV}(G S I)$ & 10 & 1.5056 & 0.5129 & 34.1 & 1.0624 & 2.0664 \\
& 15 & 1.5054 & 0.6258 & 41.0 & 1.3388 & 3.3512 \\
$\operatorname{COV}\left(m_{i}\right)$ & 12.5 & 1.5059 & 0.4948 & 32.9 & 1.0264 & 1.9290 \\
& 18.75 & 1.5056 & 0.5129 & 34.1 & 1.0624 & 2.0664 \\
$\operatorname{COV}\left(\sigma_{c}\right)$ & 12.5 & 1.5050 & 0.5426 & 36.1 & 1.1310 & 2.3422 \\
& 25 & 1.5052 & 0.3895 & 25.9 & 0.7980 & 1.1747 \\
$\operatorname{COV}(D)$ & 37.5 & 1.5056 & 0.5129 & 34.1 & 1.0624 & 2.0664 \\
& 10 & 1.5051 & 0.6700 & 44.5 & 1.4223 & 3.7569 \\
& 15 & 1.5042 & 0.5062 & 33.7 & 1.0553 & 2.0389 \\
& 1.5056 & 0.5129 & 34.1 & 1.0624 & 2.0664 \\
\end{tabular}

a

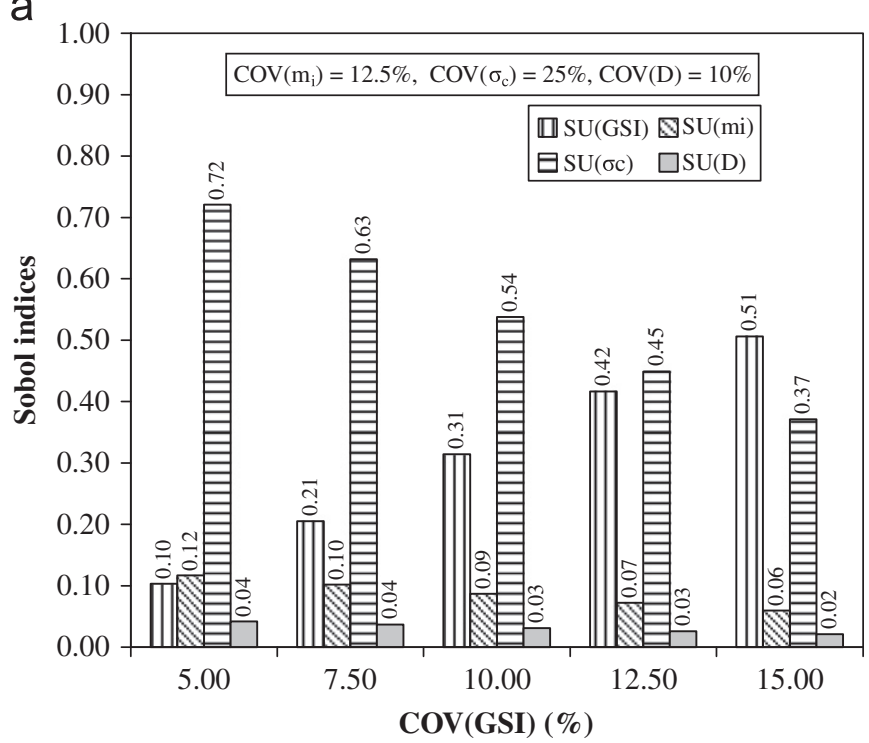

C

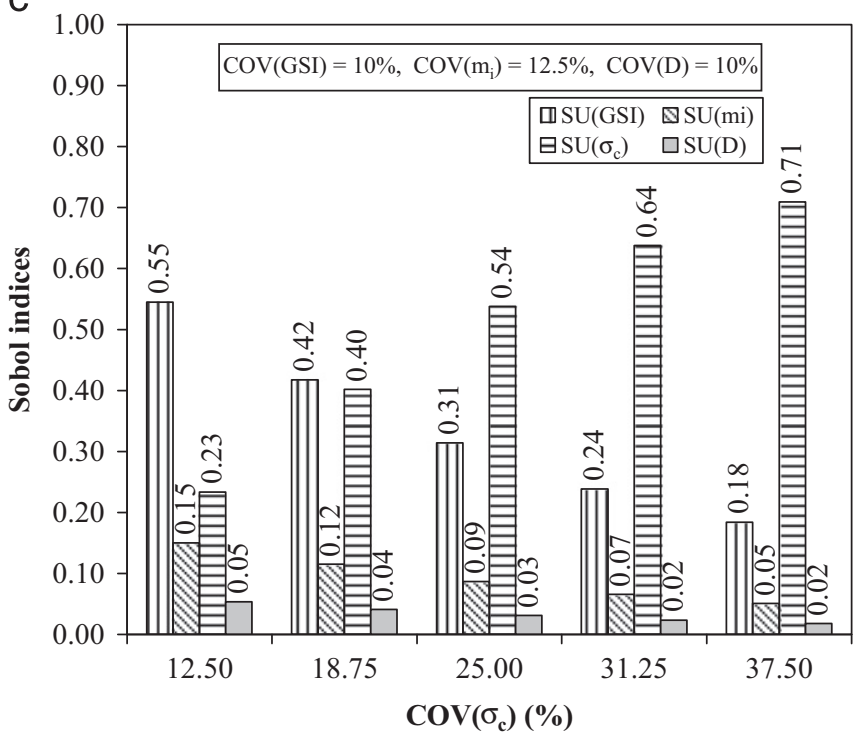

b

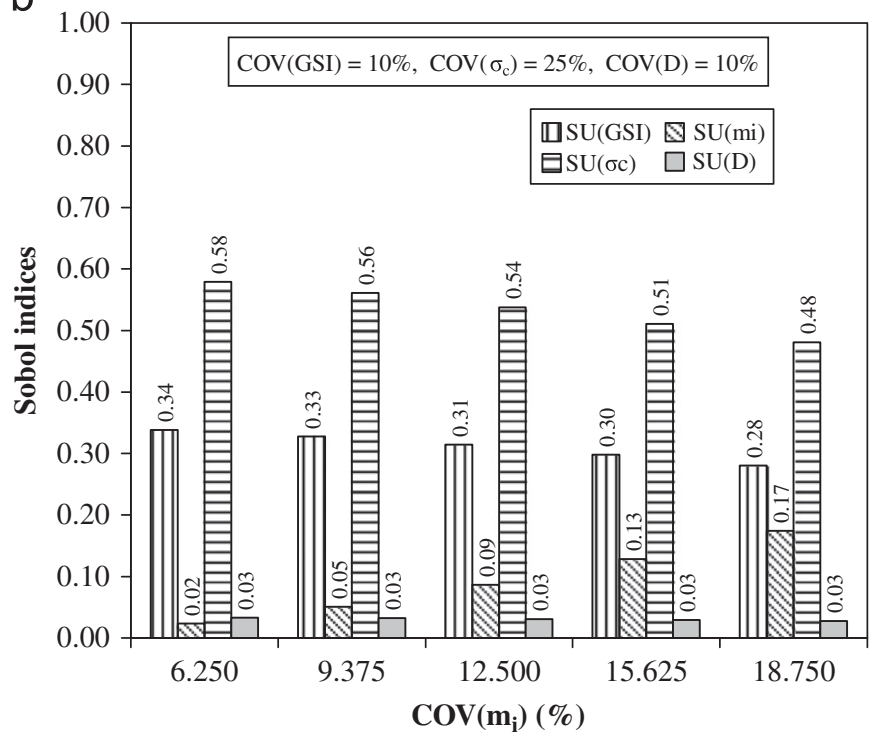

d

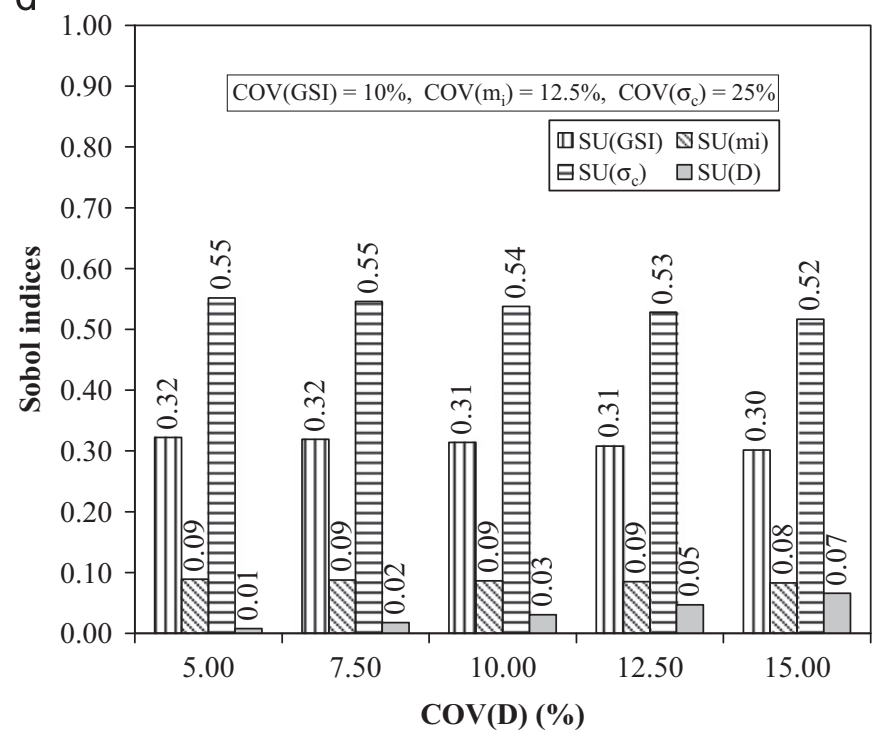

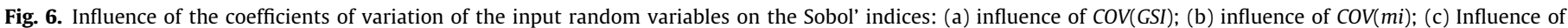
$\operatorname{COV}\left(\sigma_{c}\right)$; (d) influence of $\operatorname{COV}(D)$. 
compressive strength of the intact rock $\left(\sigma_{c}\right)$ ] to obtain reliable results of the system response.

\subsubsection{Parametric study}

The aim of this section is to study the effect of the statistical characteristics of the random variables (the coefficient of

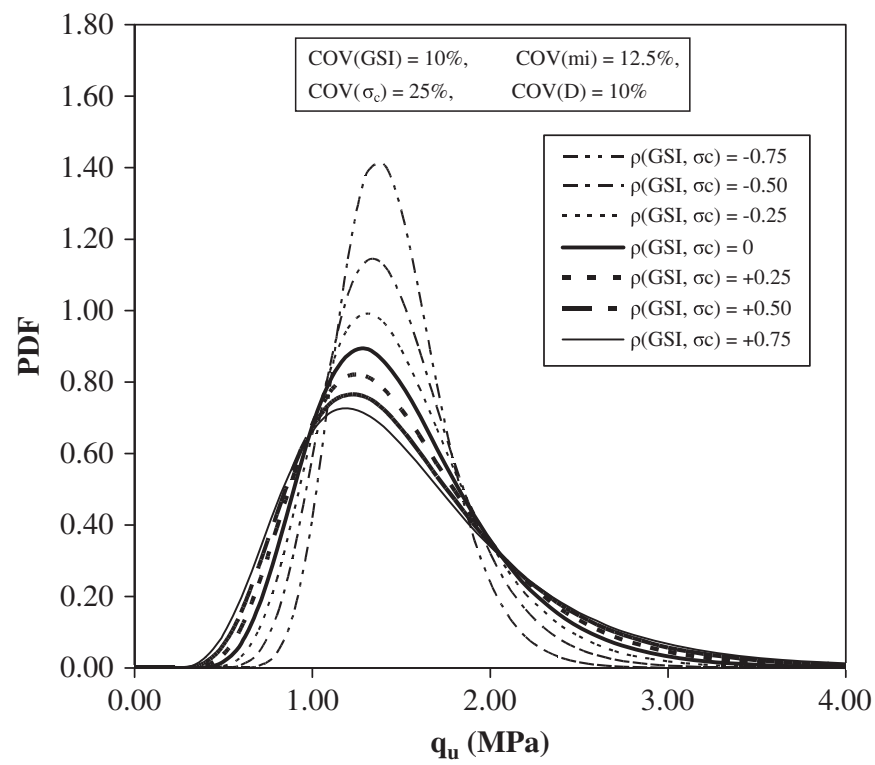

Fig. 7. Influence of the correlation coefficient $\rho\left(G S I, \sigma_{c}\right)$ on the PDF of ultimate bearing capacity.

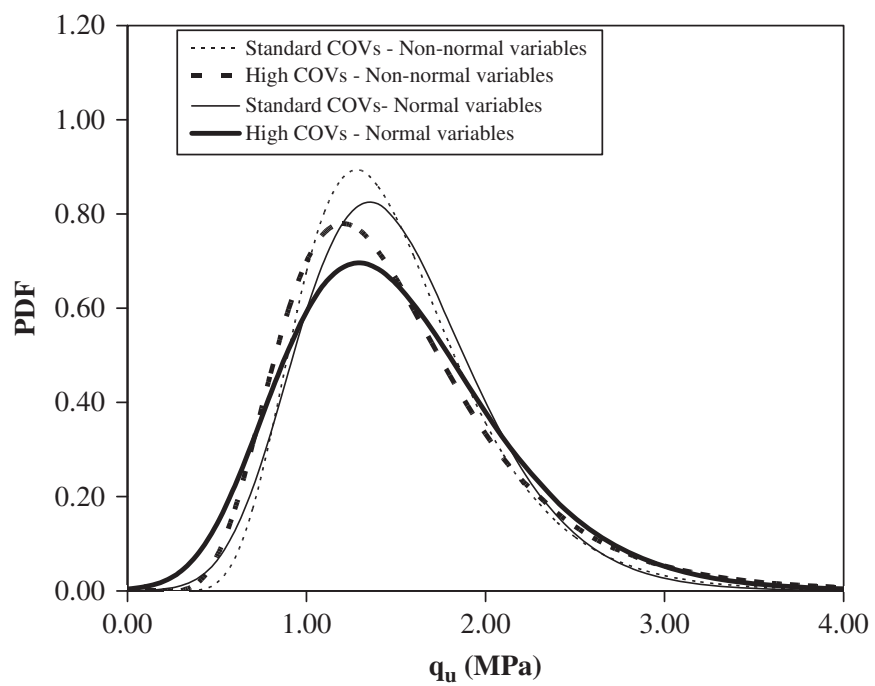

Fig. 8. Influence of the type of the probability density function of the input random variables on the PDF of the ultimate bearing capacity for two different sets of COVs. variation, the type of the probability density function and the correlation coefficient) on the statistical moments (i.e. mean $\mu$, standard deviation $\sigma$, skewness $\delta$ and kurtosis $\kappa$ ) of the ultimate bearing capacity.

4.1.2.1. Effect of the coefficients of variation of the random variables. The effect of the coefficients of variation ( $\mathrm{COV}$ ) of the random variables is studied and presented in Fig. 5 and Table 5. Figs. 5a and c show that the increase in the COV of the parameters GSI or $\sigma_{c}$ has a significant effect on the variability of the ultimate bearing capacity. On the other hand, one can see from Figs. 5b and $\mathrm{d}$ that the increase in the COV of the parameters $m_{i}$ or $D$ has practically no effect on the variability of the system response. Table 5 confirms these observations. It also shows that COV has practically no effect on the mean value of the ultimate bearing capacity. This mean value is shown to be slightly greater than the deterministic value of the ultimate bearing capacity (calculated using the mean values of the uncertain parameters). Concerning the third and fourth statistical moments, an increase in the COV of a given random variable increases the skewness and the kurtosis of the system response. Finally, the effect of COV of the random variables on the Sobol indices is shown in Fig. 6. This figure shows that the increase in $\mathrm{COV}$ of a certain variable increases its Sobol index (i.e. it increases the weight of this variable in the variability of the system response) and decreases the Sobol indices of the other variables.

4.1.2.2. Effect of the correlation coefficient and the type of the probability density function of the random variables. Fig. 7 presents the PDF of the ultimate bearing capacity for different values of the correlation coefficient $\rho\left(G S I, \sigma_{c}\right)$. This figure shows that the PDF is less spread out in the case of a negative correlation between the random variables GSI and $\sigma_{c}$. Contrarily to the case of a positive correlation (where both parameters increase or decrease together) which leads to an important variation (i.e. variability) in the ultimate bearing capacity, a negative correlation decreases the variability of the response. This is because the increase of one parameter value implies a decrease in the other parameter.

Concerning the type of the probability density function of the input random variables, two cases of normal and non-normal (log-normal) random variables combined with two configurations of COVs were considered. The "standard COVs" corresponds to the reference case presented in Table 2 [i.e. $\operatorname{COV}(G S I)=10 \%, \operatorname{COV}$ $\left(m_{i}\right)=12.5 \%, \operatorname{COV}\left(\sigma_{c}\right)=25 \%$ and $\left.\operatorname{COV}(D)=10 \%\right]$ while the "high COVs" corresponds to these values increased by $20 \%$ [i.e. COV $(G S I)=12 \%, \operatorname{COV}\left(m_{i}\right)=15 \%, \operatorname{COV}\left(\sigma_{c}\right)=30 \%$ and $\left.\operatorname{COV}(D)=12 \%\right]$. The non-normality of the input random variables has a significant influence on the shape of the PDF of the ultimate bearing capacity as can be seen from Fig. 8 for both cases of 'standard COVs' and 'high COVs'. This is confirmed by the values of the skewness and kurtosis given in Table 6. It should be emphasized, however, that the non-normality of the random variables has practically no influence on the mean and the standard deviation (and consequently on the coefficient of variation $\mathrm{COV}$ ) of the system response.

Table 6

Influence of the probability density function type of the input random variables on the statistical moments of ultimate bearing capacity for two sets of the coefficients of variation.

\begin{tabular}{lllll}
\hline & $\mu$ & $\sigma$ & COV\% & $\delta$ \\
\hline Standard COVs and non-normal variables & 1.5055 & 0.5129 & 34.1 & 1.0624 \\
Standard COVs and normal variables & 1.5056 & 0.5107 & 33.9 & 0.6292 \\
High COVs and non-normal variables & 1.5212 & 0.7380 & 48.5 & 1.5652 \\
High COVs and normal variables & 1.5203 & 0.7307 & 48.1 & 0.8755 \\
\hline
\end{tabular}


Table 7

Reliability index, design point and failure probability for different values of the applied footing load.

\begin{tabular}{|c|c|c|c|c|c|c|c|c|c|}
\hline$P_{s}(\mathrm{MN} / \mathrm{m})$ & $F$ & $\beta_{H L}$ & $G S I^{*}$ & $m_{i}^{*}$ & $\sigma_{c}^{*}(\mathrm{MPa})$ & $D^{*}$ & $P_{f}(\mathrm{FORM})$ & $P_{f}(\mathrm{MCS})$ & $\operatorname{COV}(\%)(\mathrm{MCS})$ \\
\hline 0.40 & 3.75 & 3.86 & 20.10 & 6.87 & 4.79 & 0.32 & $5.77 \times 10^{-5}$ & $5.94 \times 10^{-5}$ & 5.80 \\
\hline 0.43 & 3.50 & 3.65 & 20.32 & 6.92 & 4.98 & 0.32 & $1.32 \times 10^{-4}$ & $1.35 \times 10^{-4}$ & 3.85 \\
\hline 0.46 & 3.25 & 3.42 & 20.56 & 6.98 & 5.19 & 0.32 & $3.08 \times 10^{-4}$ & $3.06 \times 10^{-4}$ & 2.55 \\
\hline 0.50 & 3.00 & 3.18 & 20.83 & 7.04 & 5.43 & 0.32 & $7.27 \times 10^{-4}$ & $7.73 \times 10^{-4}$ & 1.61 \\
\hline 0.54 & 2.75 & 2.92 & 21.14 & 7.11 & 5.68 & 0.32 & $1.74 \times 10^{-3}$ & $1.76 \times 10^{-3}$ & 1.06 \\
\hline 0.60 & 2.50 & 2.63 & 21.47 & 7.19 & 5.99 & 0.32 & $4.21 \times 10^{-3}$ & $4.25 \times 10^{-3}$ & 0.68 \\
\hline 0.66 & 2.25 & 2.32 & 21.85 & 7.27 & 6.35 & 0.31 & $1.02 \times 10^{-2}$ & $1.03 \times 10^{-2}$ & 0.44 \\
\hline 0.74 & 2.00 & 1.96 & 22.28 & 7.37 & 6.77 & 0.31 & $2.48 \times 10^{-2}$ & $2.51 \times 10^{-2}$ & 0.28 \\
\hline 0.85 & 1.75 & 1.56 & 22.79 & 7.49 & 7.29 & 0.31 & $5.94 \times 10^{-2}$ & $5.98 \times 10^{-2}$ & 0.18 \\
\hline 0.99 & 1.50 & 1.09 & 23.39 & 7.62 & 7.94 & 0.30 & $1.37 \times 10^{-1}$ & $1.38 \times 10^{-1}$ & 0.11 \\
\hline 1.19 & 1.25 & 0.54 & 24.12 & 7.78 & 8.78 & 0.30 & $2.93 \times 10^{-1}$ & $2.95 \times 10^{-1}$ & $6.92 \times 10^{-2}$ \\
\hline 1.49 & 1.00 & -0.13 & 25.06 & 7.98 & 9.93 & 0.30 & $5.52 \times 10^{-1}$ & $5.53 \times 10^{-1}$ & $4.02 \times 10^{-2}$ \\
\hline 1.99 & 0.75 & -1.00 & 26.33 & 8.24 & 11.65 & 0.29 & $8.41 \times 10^{-1}$ & $8.42 \times 10^{-1}$ & $1.94 \times 10^{-2}$ \\
\hline 2.98 & 0.50 & -2.22 & 28.26 & 8.62 & 14.57 & 0.29 & $9.87 \times 10^{-1}$ & $9.87 \times 10^{-1}$ & $5.13 \times 10^{-3}$ \\
\hline 4.96 & 0.30 & -3.76 & 30.98 & 9.12 & 19.26 & 0.28 & 1.00 & 1.00 & $4.25 \times 10^{-4}$ \\
\hline
\end{tabular}

\subsubsection{Reliability analysis}

This section aims at performing a reliability analysis using the meta-model of the ultimate bearing capacity deduced from a PCE of order 6 . Table 7 presents the Hasofer-Lind reliability index $\beta_{H L}$, the corresponding design point $\left(G S I^{*}, m_{i}^{*}, \sigma_{c}^{*}\right.$ and $\left.D^{*}\right)$ and the probability of failure $P_{f}$ computed by FORM for different values of the applied footing load $P_{s}$. This table also presents the probability of failure $P_{f}$ computed by Monte Carlo Simulation (MCS) on the meta-model and the corresponding coefficient of variation for a number of simulations $N_{M C S}=5,000,000$ samples. All these results are presented for the case of uncorrelated and lognormal random variables. Notice that the performance function used in this section is $G=P_{u}-P_{s}$, where $P_{u}$ and $P_{s}$ are, respectively, the ultimate footing load $\left(P_{u}=q_{u} B\right)$ and the applied footing load.

From Table 7 , one can notice that the reliability index $\beta_{H L}$ decreases and consequently, the probability of failure $P_{f}$ increases, when the value of the applied footing load increases (i.e. when the safety factor $F=P_{u} / P_{s}$ decreases). When the applied footing load $P_{s}$ is equal to the ultimate footing load (i.e. $F=1$ ), the values of the random variables at the design point are very close (not exactly equal because the input random variables are non-normal) to their mean values, and the corresponding probability of failure is nearly equal to $50 \%$. In fact, the value of the design point is exactly equal to the equivalent normal mean point. Concerning the failure probability $P_{f}$ computed by FORM and MCS, Table 7 shows that as long as the failure probability is small, the corresponding coefficient of variation is important which indicates the inaccuracy of the estimated $P_{f}$ (i.e. a greater number of samples is required). However, concerning the high failure probabilities corresponding to great values of the applied footing load, they seem to be well estimated by the current MCS with a very small coefficient of variation. For the practical case $F=3$, the failure probability is equal to $7.73 \times 10^{-4}$ and the corresponding $\mathrm{COV}$ is $1.61 \%$ which is smaller than the commonly adopted value used in the literature (i.e. $10 \%$ ). Finally, notice that the failure probability computed via FORM approximation is found to have a good agreement with the one obtained from MCS for different values of the applied footing load $P_{s}$. This explains that the limit state surface in this case is almost linear around the design point, which allows one to obtain a good approximation by using FORM.

\subsubsection{Reliability-based design}

The conventional deterministic approach used in the design of a vertically loaded foundation consists in prescribing a target safety factor (generally $F=3$ ) regardless of the uncertainties involved in the input parameters. Recently, a reliability-based design (RBD) approach

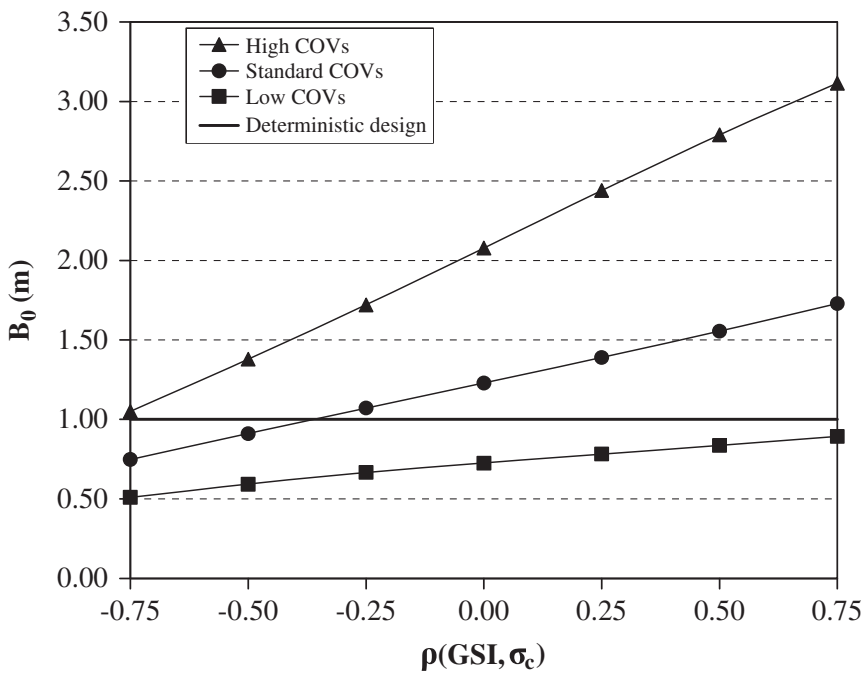

Fig. 9. Comparison between the probabilistic and the deterministic design.

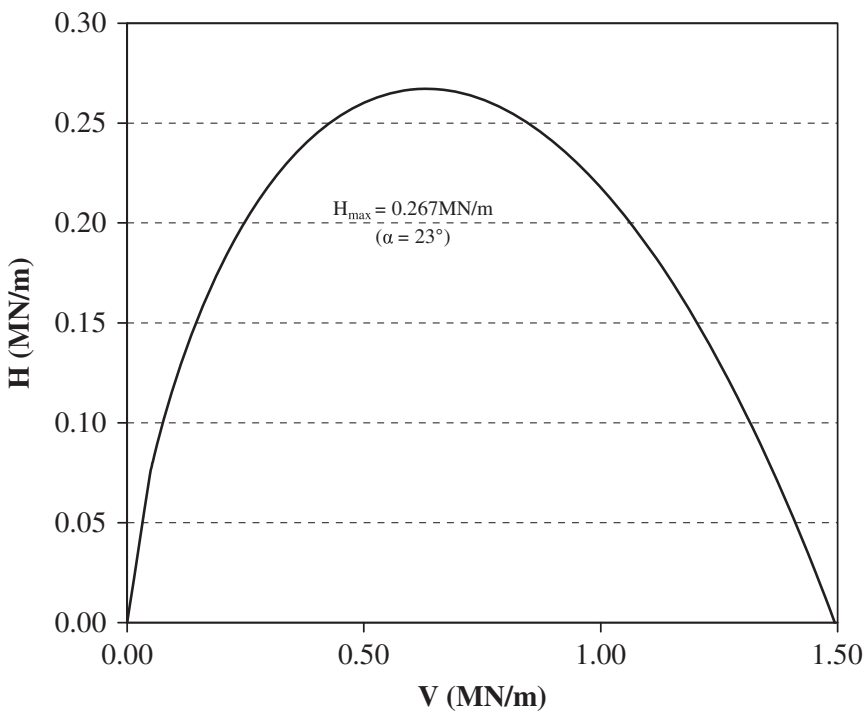

Fig. 10. Interaction diagram $(H, V)$ for the case of an inclined loading.

has been used by several authors [28 among others]. This approach was used in this section since it allows one to take into account the inherent uncertainties of the input parameters in a rational way. 
The "probabilistic foundation breadth" $B_{0}$ in this case is computed according to a target reliability index of 3.8. Note that this value is that suggested in the head Eurocode (EN 1990:2002 - Eurocode: Basis of Design) upon which Eurocode 7 and the other Eurocodes are based [29] for the ultimate limit states. The performance function used in this section is $G=q_{u}-A$ where $A$ is equal to one-third of the ultimate bearing capacity computed using the mean values of the random variables. The meta-model of the ultimate bearing capacity deduced from the PCE of order 6 is used and the Hasofer-Lind reliability index $\beta_{H L}$ is employed to compute the reliability of the foundation. For a given set of the statistical parameters of the random variables, the Hasofer-Lind reliability index is computed for different values of footing breadth by minimization with respect to the different random variables. The breadth of the footing

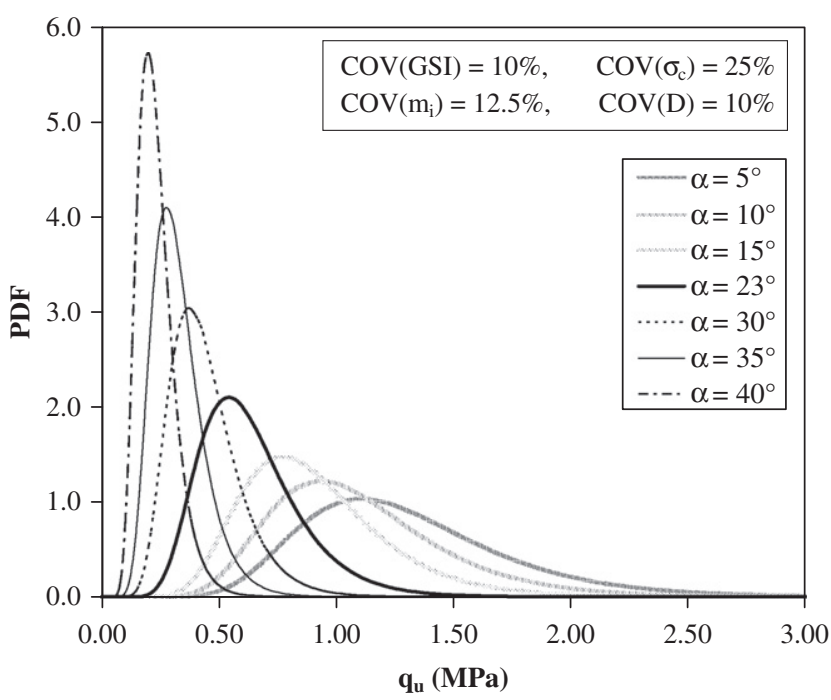

Fig. 11. PDF of the ultimate bearing capacity for different values of the footing load inclination. corresponding to a target reliability index of 3.8 is the probabilistic footing breadth.

Fig. 9 presents the probabilistic foundation breadth versus the correlation coefficient $\rho\left(G S I, \sigma_{c}\right)$ for three sets of COVs of the input random variables and for the case of non-normal variables. The "standard COVs" correspond to those presented in Table 2. The "Low COVs" and "High COVs" correspond to the values of "standard COVs" decreased or increased by $40 \%$, respectively. This figure also presents the deterministic breadth corresponding to a safety factor of 3 . The probabilistic foundation breadth increases with the increase of $\rho$ (GSI, $\sigma_{c}$ ) and the increase in the coefficients of variation of the random variables. These results allow one to observe that the deterministic footing breadth may be higher or lower than the reliability-based footing width, depending on the uncertainties of the random variables and the value of the correlation coefficient.

\subsection{Case of an obliquely loaded footing}

Fig. 10 presents the $(H, V)$ interaction diagram using the mean values of the Hoek-Brown parameters presented in Table 2. From Fig. 10 , one can observe that a maximum value of $H\left(H_{\max }=\right.$ $0.267 \mathrm{MN} / \mathrm{m}$ ) is obtained for a load inclination $\alpha=23^{\circ}$.

Fig. 11 shows the PDF of the ultimate bearing capacity for different values of the footing load inclination $\alpha$. One can see that the variability of the ultimate bearing capacity is significant in the case of a small load inclination $\alpha$ and it decreases when $\alpha$ increases. This may be explained by the fact that the response involved in the analysis is the ultimate bearing capacity. Thus, it would be logical to have the most significant variability when the punching is most predominant. Another explanation may be provided by observing the failure mechanisms shown in Fig. 12. It can be observed that the size of the failure mechanism is small in the case of high values of the load inclination $\alpha$ where the sliding is predominant. However, its size increases with the decrease in load inclination $\alpha$ where the punching is predominant. The size of the failure mechanism is maximal in the vertical load case. As expected, the ultimate bearing capacity increases with the size of the failure mechanism. Therefore, when the

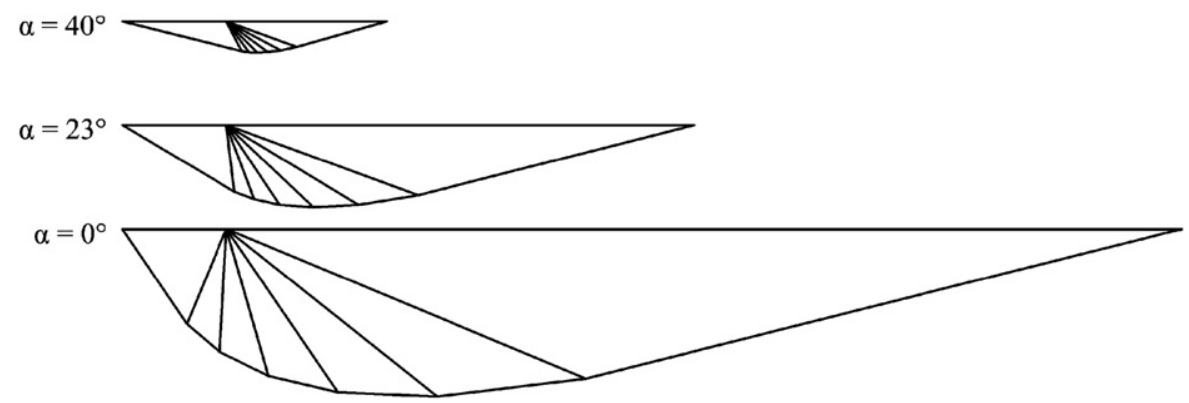

Fig. 12. Failure mechanisms for different values of the footing load inclination.

Table 8

Statistical moments of the ultimate bearing capacity for different cases of load inclination $\alpha$.

\begin{tabular}{llcccccc}
\hline $\begin{array}{l}\text { Load inclination } \\
\alpha\left({ }^{\circ}\right)\end{array}$ & 5 & 10 & 15 & 23 & 30 & 35 & \\
\hline$\mu$ & 1.3032 & 1.1004 & 0.9090 & 0.6367 & 0.4405 & 0.3260 \\
$\sigma$ & 0.4443 & 0.3758 & 0.3104 & 0.2178 & 0.1509 & 0.1119 & 0.2325 \\
COV\% & 34.1 & 34.2 & 34.2 & 34.2 & 34.3 & 34.3 & 34.4 \\
$\kappa$ & 1.0627 & 1.0672 & 1.0693 & 1.0698 & 1.0709 & 1.0712 & 1.0742 \\
$\delta$ & 2.0710 & 2.0928 & 2.1113 & 2.0969 & 2.1051 & 2.095 \\
\hline
\end{tabular}


failure mechanism is small (i.e. the sliding is predominant), the variation in the modified Hoek-Brown parameters (GSI, $m_{i}, \sigma_{c}$ and $D$ ) does not have a significant effect on the ultimate bearing capacity. However, when this mechanism is large (i.e. the punching is predominant) a small variation in the modified HoekBrown parameters (GSI, $m_{i}, \sigma_{c}$ and $D$ ) results in a significant effect on the ultimate bearing capacity. Finally, it has to be noted that since the coefficient of variation and the correlation coefficient of the input random variables have not been changed for the different cases of the footing load inclination $\alpha$, the COVs of the corresponding model response (i.e. ultimate bearing capacity) are not changed either (except for its mean and standard deviation, of course). This can be easily observed from Table 8 .

\section{Conclusion}

A probabilistic analysis of a centrally loaded strip footing resting on a rock mass has been performed. The case where the strength of the rock material can be described by the modified Hoek-Brown failure criterion was considered in the analysis. The deterministic models were based on the kinematical approach of the limit analysis theory. The polynomial chaos expansion (PCE) methodology was used for the probabilistic and reliability-based analysis. The uncertain parameters involved in the analysis were the geological strength index (GSI), the uniaxial compressive strength of the intact rock $\left(\sigma_{c}\right)$, the intact rock material constant $\left(m_{i}\right)$ and the disturbance coefficient $(D)$. For the vertically loaded footing, it was shown that:

(a) The variability of the ultimate bearing capacity increases with the increase in the coefficients of variation of the random variables; GSI and $\sigma_{c}$ being of greater effect.

(b) The assumption of negatively correlated variables was found to give less spread out PDF of the ultimate bearing capacity with respect to the case of uncorrelated variables.

(c) The non-normality of the input random variables has a significant impact on the shape of the PDF of the ultimate bearing capacity. There is an almost no effect on the mean and standard deviation of the system response and consequently, no effect on the coefficient of variation of this response.

(d) The increase in the COV of a certain variable increases its Sobol index (i.e. it increases its weight in the variability of the system response) and decreases the Sobol indices of the other variables.

(e) The failure probability computed by FORM approximation for different values of the punching safety factor has shown a good agreement with the one obtained from Monte Carlo simulation. This observation may indicate that the limit state surface is almost linear around the design point.

(f) The probabilistic footing breadth based on a reliability-based design (RBD) may be greater or smaller than the deterministic breadth depending on the values of the input statistical parameters.

(g) Finally, in the inclined load case, it was found that the variability of the ultimate bearing capacity decreases with the increase of the footing load inclination. However, the coefficient of variation of this ultimate bearing capacity is constant regardless of the footing load inclination.

\section{Appendix A. M1 Mechanism}

For a triangular block $i$, the lengths $l_{i}$ and $d_{i}$, and the surface $S_{i}$ are given as follows:

$l_{i}=\frac{B_{0}}{2 \cos (\theta)} \prod_{j=1}^{i-1} \frac{\sin \beta_{j}}{\sin \left(\alpha_{j}+\beta_{j}\right)}$

$d_{i}=\frac{B_{0}}{2 \cos (\theta)} \frac{\sin \alpha_{i}}{\sin \left(\alpha_{i}+\beta_{i}\right)} \prod_{j=1}^{i-1} \frac{\sin \beta_{j}}{\sin \left(\alpha_{j}+\beta_{j}\right)}$

$S_{i}=\frac{B_{0}^{2}}{2} \frac{\sin \alpha_{i} \sin \beta_{j}}{4 \cos ^{2} \theta \sin \left(\alpha_{i}+\beta_{i}\right)} \prod_{j=1}^{i-1} \frac{\sin ^{2} \beta_{j}}{\sin ^{2}\left(\alpha_{j}+\beta_{j}\right)}$

The expressions of $N_{\gamma}, N_{q}$ and $N_{\sigma_{c}}$ in Eq. (22) are given as follows:

$$
\begin{aligned}
N_{\gamma}= & \frac{\tan (\theta)}{2}+\frac{\cos \left(\theta-\varphi_{0,1}\right)}{2 \cos ^{2}(\theta) \sin \left(\beta_{1}-\varphi_{1}-\varphi_{0,1}\right)} \\
& \times \sum_{i=1}^{k}\left[\frac{\sin \left(\alpha_{i}\right) \sin \left(\beta_{i}\right)}{\sin \left(\alpha_{i}+\beta_{i}\right)} \sin \left(\beta_{i}-\theta-\sum_{j=1}^{i-1} \alpha_{j}-\varphi_{i}\right)\right. \\
& \left.\times \prod_{j=1}^{i-1} \frac{\sin \left(\alpha_{j}+\beta_{j}-\varphi_{j}-\varphi_{j, j+1}\right)}{\sin \left(\beta_{j+1}-\varphi_{j+1}-\varphi_{j, j+1}\right) \sin ^{2}\left(\alpha_{j}+\beta_{j}\right)}\right] \\
N_{q}= & \frac{\cos \left(\theta-\varphi_{0,1}\right) \sin \left(\beta_{k}-\theta-\sum_{j=1}^{k-1} \alpha_{j}-\varphi_{k-1, k}\right) \sin \left(\beta_{k}\right)}{\cos (\theta) \sin \left(\beta_{k}-\varphi_{k}-\varphi_{k-1, k}\right) \sin \left(\alpha_{k}+\beta_{k}\right)} \\
& \times \prod_{j=1}^{k-1} \frac{\sin \left(\beta_{i}\right) \sin \left(\alpha_{j}+\beta_{j}-\varphi_{j}-\varphi_{j, j+1}\right)}{\sin \left(\beta_{j}-\varphi_{j}-\varphi_{j-1, j}\right) \sin \left(\alpha_{j}+\beta_{j}\right)}
\end{aligned}
$$

$$
\begin{aligned}
N_{\sigma_{c}} & =\frac{1}{\cos (\theta)} \\
& {\left.\left[\frac{s}{m} \frac{v_{0,1}^{(n)}}{v_{0}}+\left(n^{(n /(1-n))}-n^{(1 /(1-n))}\right) m^{(n /(1-n))}\left[\frac{v_{0}}{v_{0,1}^{(n)}} \frac{v_{0,1}-v_{0,1}^{(n)}}{2 v_{0}}\right)^{1 / n}\right]^{(n /(1-n))}\right] } \\
& +\sum_{i=1}^{k-1}\left[\frac{s}{m} \frac{v_{i, i+1}^{(n)}}{v_{0}}+\left(n^{(n /(1-n))}-n^{(1 /(1-n))}\right) m^{(n /(1-n))}\right. \\
& {\left.\left.\left[\frac{v_{0}}{v_{i, i+1}^{(n)}} \frac{v_{i, i+1}-v_{i, i+1}^{(n)}}{2 v_{0}}\right)^{1 / n}\right]^{(n /(1-n))}\right] \times \frac{1}{\cos (\theta)} \prod_{j=1}^{i} \frac{\sin \left(\beta_{j}\right)}{\sin \left(\alpha_{j}+\beta_{j}\right)} } \\
& +\sum_{i=1}^{k} 2 \frac{v_{i}}{v_{0}} \sin \left(\varphi_{i}\right) \frac{d_{i}}{B_{0}} \\
& {\left[\frac{s}{m}+\left(n^{(n /(1-n))}-n^{(1 /(1-n))}\right) m^{(n /(1-n))}\left(\frac{1-\sin \left(\varphi_{i}\right)}{2 \sin \left(\varphi_{i}\right)}\right)^{(1 /(1-n))}\right] }
\end{aligned}
$$




\section{Appendix B. M2 Mechanism}

For a triangular block $i$, the lengths $l_{i}$ and $d_{i}$, and the surface $S_{i}$ are given as follows:

$l_{i}=B_{0} \frac{\sin \beta_{1}}{\sin \left(\alpha_{1}+\beta_{1}\right)} \prod_{j=2}^{i} \frac{\sin \beta_{j}}{\sin \left(\alpha_{j}+\beta_{j}\right)}$

$d_{i}=B_{0} \frac{\sin \beta_{1}}{\sin \left(\alpha_{1}+\beta_{1}\right)} \frac{\sin \alpha_{i}}{\sin \beta_{i}} \prod_{j=2}^{i} \frac{\sin \beta_{j}}{\sin \left(\alpha_{j}+\beta_{j}\right)}$

$\left.G 6=\sum_{i=1}^{k-1}\left[\begin{array}{l}\frac{s}{m} \frac{v_{i, i+1}^{(n)}}{v_{1}} \frac{\sin \beta_{1}}{\sin \left(\alpha_{1}+\beta_{1}\right)} \prod_{j=2}^{i} \frac{\sin \beta_{j}}{\sin \left(\alpha_{j}+\beta_{j}\right)} \\ +\left(n^{n /(1-n)}-n^{1 /(1-n)}\right) m^{n /(1-n)}\left[\frac{v_{1}}{v_{i, i+1}^{(n)}} \frac{v_{i, i+1}-v_{i, i+1}^{(n)}}{2 v_{1}}\right)^{1 / n}\end{array}\right]^{n /(1-n)} \frac{\sin \beta_{1}}{\sin \left(\alpha_{1}+\beta_{1}\right)} \prod_{j=2}^{i} \frac{\sin \beta_{j}}{\sin \left(\alpha_{j}+\beta_{j}\right)}\right]$
$G 5=\sum_{i=1}^{k}\left[\frac{s}{m} \frac{v_{i}^{(n)}}{v_{1}} \frac{\sin \alpha_{i}}{\sin \beta_{i}} \frac{\sin \beta_{1}}{\sin \left(\alpha_{1}+\beta_{1}\right)} \prod_{j=2}^{i} \frac{\sin \beta_{j}}{\sin \left(\alpha_{j}+\beta_{j}\right)}\right.$

$\left.+\left(n^{n /(1-n)}-n^{1 /(1-n)}\right) m^{n /(1-n)}\left[\frac{v_{1}}{v_{i, i+1}^{(n)}} \frac{v_{i, i+1}-v_{i, i+1}^{(n)}}{2 v_{1}}\right)^{1 / n}\right]^{n /(1-n)}$

$\left.\frac{\sin \alpha_{i}}{\sin \beta_{i}} \frac{\sin \beta_{1}}{\sin \left(\alpha_{1}+\beta_{1}\right)} \prod_{j=2}^{i} \frac{\sin \beta_{j}}{\sin \left(\alpha_{j}+\beta_{j}\right)}\right]$
$S_{i}=\frac{B_{0}^{2}}{2} \frac{\sin ^{2} \beta_{1}}{\sin ^{2}\left(\alpha_{1}+\beta_{1}\right)} \frac{\sin \alpha_{i} \sin \left(\alpha_{i}+\beta_{i}\right)}{\sin \beta_{j}} \prod_{j=2}^{i} \frac{\sin ^{2} \beta_{j}}{\sin ^{2}\left(\alpha_{j}+\beta_{j}\right)}$

The expressions of $N_{\gamma}, N_{q}$ and $N_{\sigma_{c}}$ in Eq. (22) are given as follows:

$$
\begin{aligned}
& N_{\gamma}=\frac{-1}{\sin \left(\beta_{1}-\varphi_{1}\right)+\tan \alpha \cos \left(\beta_{1}-\varphi_{1}\right)}(G 1+\tan \alpha . G 2) \\
& N_{q}=\frac{-1}{\sin \left(\beta_{1}-\varphi_{1}\right)+\tan \alpha \cos \left(\beta_{1}-\varphi_{1}\right)}(G 3+\tan \alpha . G 4) \\
& N_{\sigma_{c}}=\frac{1}{\sin \left(\beta_{1}-\varphi_{1}\right)+\tan \alpha \cos \left(\beta_{1}-\varphi_{1}\right)}(G 5+\tan \alpha . G 6)
\end{aligned}
$$

where

$$
G 1=\frac{\sin ^{2} \beta_{1}}{\sin ^{2}\left(\alpha_{1}+\beta_{1}\right)} \sum_{i=1}^{k}\left[\begin{array}{l}
\frac{\sin \alpha_{i} \sin \left(\alpha_{i}+\beta_{i}\right)}{\sin \beta_{i}} \sin \left(\beta_{i}-\varphi_{i}-\sum_{j=1}^{i-1} \alpha_{j}\right) \\
\times \prod_{j=2}^{i} \frac{\sin ^{2} \beta_{j}}{\sin ^{2}\left(\alpha_{j}+\beta_{j}\right)} \prod_{j=1}^{i-1} \frac{\sin \left(\alpha_{j}+\beta_{j}-\varphi_{j}-\varphi_{j-1, j}\right)}{\sin \left(\beta_{j+1}-\varphi_{j+1}-\varphi_{j-1, j}\right)}
\end{array}\right]
$$

$G 2=\frac{\sin ^{2} \beta_{1}}{\sin ^{2}\left(\alpha_{1}+\beta_{1}\right)} \sum_{i=1}^{k}\left[\begin{array}{l}\frac{\sin \alpha_{i} \sin \left(\alpha_{i}+\beta_{i}\right)}{\sin \beta_{i}} \cos \left(\beta_{i}-\varphi_{i}-\sum_{j=1}^{i-1} \alpha_{j}\right) \\ \times \prod_{j=2}^{i} \frac{\sin ^{2} \beta_{j}}{\sin ^{2}\left(\alpha_{j}+\beta_{j}\right)} \prod_{j=1}^{i-1} \frac{\sin \left(\alpha_{j}+\beta_{j}-\varphi_{j}-\varphi_{j-1, j}\right)}{\sin \left(\beta_{j+1}-\varphi_{j+1}-\varphi_{j-1, j}\right)}\end{array}\right]$

$$
\begin{aligned}
G 3= & \frac{\sin \beta_{1}}{\sin \left(\alpha_{1}+\beta_{1}\right)} \sin \left(\beta_{k}-\varphi_{k}-\sum_{j=1}^{k-1} \alpha_{j}\right) \\
& \times \prod_{j=2}^{k} \frac{\sin \beta_{j}}{\sin \left(\alpha_{j}+\beta_{j}\right)} \prod_{j=1}^{k-1} \frac{\sin \left(\alpha_{j}+\beta_{j}-\varphi_{j}-\varphi_{j-1, j}\right)}{\sin \left(\beta_{j+1}-\varphi_{j+1}-\varphi_{j-1, j}\right)} \\
G 4= & \frac{\sin \beta_{1}}{\sin \left(\alpha_{1}+\beta_{1}\right)} \cos \left(\beta_{k}-\varphi_{k}-\sum_{j=1}^{k-1} \alpha_{j}\right)
\end{aligned}
$$

\section{References}

[1] Soubra AH. Upper-bound solutions for bearing capacity of foundations. J Geotech Geoenviron Eng ASCE 1999;125(1):59-68.

[2] Maghous S, de Buhan P, Bekaert A. Failure design of jointed rock structures by means of a homogenization approach. Mech Cohesive-Frictional Mater 1998;3:207-28.

[3] Yang XL, Yin JH. Upper bound solution for ultimate bearing capacity with a modified Hoek-Brown failure criterion. Int J Rock Mech Min Sci 2005;42: 550-60.

[4] Saada Z, Maghous S, Garnier D. Bearing capacity of shallow foundations on rocks obeying a modified Hoek-Brown failure criterion. Comput Geotech 2008;35:144-54.

[5] Griffiths DV, Fenton GA. Bearing capacity of spatially random soil: the undrained clay Prandtl problem revisited. Géotechnique 2001;51(4):351-9.

[6] Fenton GA, Griffiths DV. Bearing-capacity prediction of spatially random $c-\varphi$ soils. Can Geotech J 2003;40:64-5.

[7] Przewlocki J. A stochastic approach to the problem of bearing capacity by the method of characteristics. Comput Geotech 2005;32:370-6.

[8] Popescu R, Deodatis G, Nobahar A. Effects of random heterogeneity of soil properties on bearing capacity. Probab Eng Mech 2005;20:324-41.

[9] Youssef Abdel Massih DS, Soubra AH, Low BK. Reliability-based analysis and design of strip footings against bearing capacity failure. J Geotech Geoenviron Eng ASCE 2008;134(7):917-28.

[10] Youssef Abdel Massih DS, Soubra AH. Reliability-based analysis of strip footings using response surface methodology. Int J Geomech ASCE 2008;8(2): 134-43.

[11] Soubra AH, Youssef Abdel Massih DS. Probabilistic analysis and design at the ultimate limit state of obliquely loaded strip footings. Géotechnique 2010;60(4):275-85.

[12] Cho SE, Park HC. Effect of spatial variability of cross-correlated soil properties on bearing capacity of strip footing. Int J Numer Anal Meth Geomech 2010;34:1-26.

[13] Isukapalli SS, Roy A, Georgopoulos PG. Stochastic response surface methods (SRSMs) for uncertainty propagation: application to environmental and biological systems. Risk Anal 1998;18(3):357-63.

[14] Phoon KK, Haung SP. Geotechnical probabilistic analysis using collocationbased stochastic response surface method. In: Kanda J, Takada T, Furuta H, editors. Applications of statistics and probability in civil engineering, Tokyo, 2007.

[15] Huang SP, Liang B, Phoon KK. Geotechnical probabilistic analysis by collocation-based stochastic response surface method: an EXCEL add-in implementation. Georisk 2009;3(2):75-86.

[16] Isukapalli SS. An uncertainty analysis of transport-transformation models. PhD thesis, Rutgers Univ, New Jersey, 1999.

[17] Xiu D, Karniadakis GE. The Wiener-Askey polynomial chaos for stochastic differential equations. J Sci Comput 2002;24(2):619-44.

[18] Webster M, Tatang M, McRae G. Application of the probabilistic collocation method for an uncertainty analysis of a simple ocean model. Technical report, MIT joint program on the science and policy of global change, Reports series no. 4. Cambridge, MA: MIT; 1996. 
[19] Sudret B. Global sensitivity analysis using polynomial chaos expansion. Reliab Eng System Saf 2008;93:964-79.

[20] Blatman G, Sudret B. An adaptive algorithm to build up sparse polynomial chaos expansions for stochastic finite element analysis. Probab Eng Mech 2010;25:183-97.

[21] Hoek E, Brown E. Empirical strength criterion for rock masses. J Geotech Eng Div 1980;106(GT9):1013-35.

[22] Hoek E, Marinos P. A brief history of the development of the Hoek-Brown failure criterion. Soils Rocks 2007;30(2):85-92.

[23] Brown ET. Estimating the mechanical properties of rock masses. In: Potvin Y, Carter J, Dyskin A, Jeffrey R, Editors. Proc 1st southern hemisphere int rock mech symp, Perth, vol. 1; 2008, pp. 3-21.

[24] Hoek E, Carranze-Torres C, Corkum B. Hoek-Brown failure criterion-2002 edition. In: Proc North Am rock mech symp, Toronto, July 2002, p. 267-73.
[25] Hoek E, Brown ET. Practical estimates of rock mass strength. Int J Rock Mech Min Sci 1997;34(8):1165-86.

[26] Collins IF, Gunn CIM, Pender MJ, Yan W. Slope stability analyses for materials with a nonlinear failure envelope. Int J Num Anal Meth Geomech 1988;12: 533-50.

[27] Hoek E. Reliability of Hoek-Brown estimates of rock mass properties and their impact on design. Technical note. Int J Rock Mech Min Sci 1998;35: 63-8.

[28] Phoon KK, Kulhawy FH, Grigoriu MD. Multiple resistance factor design for shallow transmission line structure foundations. J Geotech Geoenviron Eng 2003;129:807-18.

[29] Orr TL, Breysse D. Eurocode 7 and reliability based design. In: Phoon KK editor. Reliability-based design in geotechnical engineering: computations and applications. London: Taylor \& Francis; 2008 [Chapter8]. 